\title{
UNCERTAINTY QUANTIFICATION FOR PDEs WITH ANISOTROPIC RANDOM DIFFUSION*
}

\author{
H. HARBRECHT ${ }^{\dagger}$, M. D. PETERS ${ }^{\dagger}$, AND M. SCHMIDLIN $^{\dagger}$
}

\begin{abstract}
In this article, we consider elliptic diffusion problems with an anisotropic random diffusion coefficient. We model the notable direction in terms of a random vector field and derive regularity results for the solution's dependence on the random parameter. It turns out that the decay of the vector field's Karhunen-Loève expansion entirely determines this regularity. The obtained results allow for sophisticated quadrature methods, such as the quasi-Monte Carlo method or the anisotropic sparse grid quadrature, in order to approximate quantities of interest, like the solution's mean or the variance. Numerical examples in three spatial dimensions are provided to supplement the presented theory.
\end{abstract}

Key words. uncertainty quantification, anisotropic diffusion, regularity estimates

AMS subject classifications. 35R60, 65N30,60H35

DOI. $10.1137 / 16 \mathrm{M} 1085760$

1. Introduction. Many phenomena in science and engineering are modeled as boundary value problems for an unknown function. Because, in general, the computation of an exact solution of such a boundary value problem is infeasible, it is necessary to use numerical schemes that yield approximations of the solution. When using numerical methods, such as finite elements or finite differences, the behavior of these numerical simulations is generally well understood for input data, such as boundary values or coefficients, that are given exactly. However, for many applications, the input data are not known exactly and can be thought of as being subject to uncertainty, for example, when they are based on measurements. This then implies that the solution of the boundary value problem is also subject to uncertainty.

Specifically, let us consider the second order diffusion problem where uncertainty regarding the diffusion coefficient $\mathbf{A}$ has been accounted for by considering it as a random matrix field over a given probability space $(\Omega, \mathcal{F}, \mathbb{P})$, i.e.,

$$
\text { for almost every } \omega \in \Omega:\left\{\begin{aligned}
-\operatorname{div}_{\mathbf{x}}\left(\mathbf{A}(\omega) \nabla_{\mathbf{x}} u(\omega)\right)=f & & \text { in } D, \\
u(\omega)=0 & & \text { on } \Gamma_{D}, \\
\left\langle\mathbf{A}(\omega) \nabla_{\mathbf{x}} u(\omega), \mathbf{n}\right\rangle=g & & \text { on } \Gamma_{N},
\end{aligned}\right.
$$

on a domain $D$ given a mixed boundary condition, where the function $f$ describes the known source and the function $g$ the conormal derivative at the Neumann boundary.

We note that the numerical treatment of isotropic random diffusion coefficients, i.e., $\mathbf{A}(\omega)=a(\omega) \mathbf{I}$, has already been considered, for example, in $[3,4,6,8,18]$. However, since the simulations in applications may require anisotropic diffusion, we subsequently consider anisotropic random diffusion coefficients that fit the following

${ }^{*}$ Received by the editors July 19, 2016; accepted for publication (in revised form) December 29, 2016; published electronically April 25, 2017.

http://www.siam.org/journals/sinum/55-2/M108576.html

Funding: The work of the authors was supported by the Swiss National Science Foundation (SNSF) through the project "Multilevel Methods and Uncertainty Quantification in Cardiac Electrophysiology" (grant 205321_169599).

${ }^{\dagger}$ University of Basel, Department of Mathematics and Computer Science, Spiegelgasse 1, 4051 Basel, Switzerland (helmut.harbrecht@unibas.ch, michael.peters@unibas.ch, marc.schmidlin@ unibas.ch). 


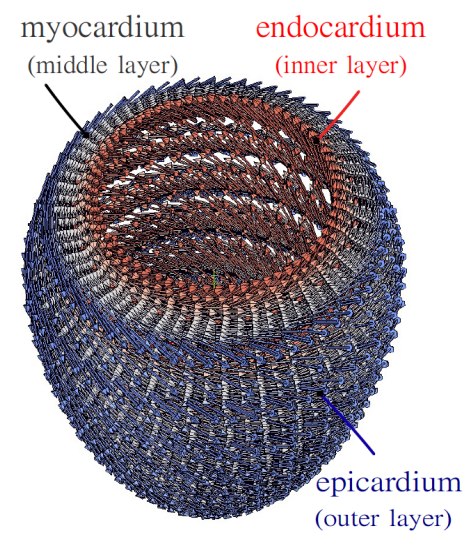

FIG. 1. Fiber orientation in the left ventricle.

description: The anisotropic random diffusion coefficient has, at every point in $D$, a notable direction regarding diffusion; that is, a direction perpendicular to which diffusion is isotropic with a global strength $a$ and in which the strength of diffusion may be considerably different and also may vary in space. We can represent this notable direction with its spatially varying directional strength as a random vector field $\mathbf{V}$.

One interpretation for random diffusion coefficients of this type is to consider diffusion in a medium that is comprised of thin fibers. The direction of these fibers is then described by the direction of $\mathbf{V}$ and the strength of diffusion in a fiber is given by $\|\mathbf{V}\|_{2}$. The diffusion from a fiber to a neighboring fiber is the diffusion that is perpendicular to $\mathbf{V}$ and is thus considered to have strength $a$.

The application we have in mind here amounts from cardiac electrophysiology: The electrical activation of the human heart has been an active area of research during the last decades. It has been known for a long time that the heart exhibits a fibrous structure. By now, it is well understood that these fibers play a major role for both the electrical and the mechanical properties of the heart muscle. More precisely, the fibers have a very complex but also well-organized structure, exhibiting key features that can be identified in all healthy subjects, such as a helical distribution with opposite orientations, from the endocardium to the epicardium, see, e.g., [19, 20]. A visualization of the fiber structure can be found in Figure $1 .{ }^{1}$ in particular, for visualizations of the cardiac fiber structure. However, the exact fiber dislocations vary between different individuals and may also change over time within a single individual due to pathologies, such as infarctions. Then, the fiber structure is perturbed with the introduction of high variability areas in the presence of scars. This uncertainty in the fiber direction is modeled via the vector field $\mathbf{V}$. In this particular application, the ratio between $\|\mathbf{V}\|_{2}$ and $a$ is typically of the order ten to one; see, e.g., [9, 20].

Moreover, random diffusion coefficients of this type may also be used to model the diffusion in a laminar medium, i.e., a medium comprised of stacked thin layers, by choosing the direction of $\mathbf{V}$ as the normals on the layers. Then, we have that in a layer, the diffusion is isotropic with the strength $a$, and in-between layers, that is, in the direction given by $\mathbf{V}$, the diffusion strength is given by $\|\mathbf{V}\|_{2}$.

\footnotetext{
${ }^{1}$ We have to thank the research group of Prof. Dr. Rolf Krause from the Institute of Computational Science, Università della Svizzera Italiana, Lugano, Switzerland and in particular Sonia Pozzi for providing us with this visualization.
} 
The sections hereafter are organized as follows: In section 2, we provide basic definitions and notation concerning the functional analytic framework. Therafter, we introduce the model problem which especially includes the formula that expresses the diffusion coefficient $\mathbf{A}$ in terms of $\mathbf{V}$ and $a$. Then, in section 3 , we reformulate the model problem into a stochastically parametric and spatially weak formulation, by using the Karhunen-Loève expansion of the diffusion describing field $\mathbf{V}$ to arrive at a stochastically parametrized form of the diffusion coefficient $\mathbf{A}$. This also enables us to conclude the well-posedness of the model problem. Section 4 is dedicated to the regularity of the solution of the model problem with respect to the random parameter from the Karhunen-Loève expansion of the diffusion describing field V. This regularity then yields convergence rates, when considering quasi-Monte Carlo or anisotropic sparse grid quadrature to calculate the solution's mean and variance. Numerical examples are then provided in section 5 as validation. Last, our conclusions are given in section 6 .

\section{Problem formulation.}

2.1. Notation and precursory remarks. For a given Banach space $\mathcal{X}$ and a complete measure space $\mathcal{M}$ with measure $\mu$ the space $L_{\mu}^{p}(\mathcal{M} ; \mathcal{X})$ for $1 \leq p \leq \infty$ denotes the Bochner space (see [15]), which contains all equivalence classes of strongly measurable functions $v: \mathcal{M} \rightarrow \mathcal{X}$ with finite norm

$$
\|v\|_{L_{\mu}^{p}(\mathcal{M} ; \mathcal{X})}:= \begin{cases}{\left[\int_{\mathcal{M}}\|v(x)\|_{\mathcal{X}}^{p} \mathrm{~d} \mu(x)\right]^{1 / p},} & p<\infty, \\ \underset{x \in \mathcal{M}}{\operatorname{ess} \sup }\|v(x)\|_{\mathcal{X}}, & p=\infty .\end{cases}
$$

A function $v: \mathcal{M} \rightarrow \mathcal{X}$ is strongly measurable if there exists a sequence of countably valued measurable functions $v_{n}: \mathcal{M} \rightarrow \mathcal{X}$, such that for almost every $m \in \mathcal{M}$ we have $\lim _{n \rightarrow \infty} v_{n}(m)=v(m)$. Note that, for finite measures $\mu$, we also have the usual inclusion $L_{\mu}^{p}(\mathcal{M} ; \mathcal{X}) \supset L_{\mu}^{q}(\mathcal{M} ; \mathcal{X})$ for $1 \leq p<q \leq \infty$.

When $\mathcal{X}$ is a separable Hilbert space and $\mathcal{M}$ is a separable measure space, the Bochner space $L_{\mu}^{2}(\mathcal{M} ; \mathcal{X})$ is also a separable Hilbert space with the inner product

$$
(u, v)_{L_{\mu}^{2}(\mathcal{M} ; \mathcal{X})}:=\int_{\mathcal{M}}(u(x), v(x))_{\mathcal{X}} \mathrm{d} \mu(x)
$$

and is isomorphic to the tensor product space $L_{\mu}^{2}(\mathcal{M}) \otimes \mathcal{X}$; see [16].

Subsequently, we will always equip $\mathbb{R}^{d}$ with the norm $\|\cdot\|_{2}$ induced by the canonical inner product $\langle\cdot, \cdot\rangle$ and $\mathbb{R}^{d \times d}$ with the norm $\|\cdot\|_{F}$ induced by the Frobenius inner product $\langle\cdot, \cdot\rangle_{F}$. Then, for $\mathbf{v}, \mathbf{w} \in \mathbb{R}^{d}$, the Cauchy-Schwartz inequality gives us

$$
\left|\mathbf{v}^{\top} \mathbf{w}\right|=|\langle\mathbf{v}, \mathbf{w}\rangle| \leq\|\mathbf{v}\|_{2}\|\mathbf{w}\|_{2} \quad \text { and the special case } \quad \mathbf{v}^{\top} \mathbf{v}=\langle\mathbf{v}, \mathbf{v}\rangle=\|\mathbf{v}\|_{2}^{2},
$$

and we also have, by straightforward computation, that $\left\|\mathbf{v w}^{\top}\right\|_{F}=\|\mathbf{v}\|_{2}\|\mathbf{w}\|_{2}$.

2.2. The model problem. Let $(\Omega, \mathcal{F}, \mathbb{P})$ be a separable, complete probability space. We consider the following second order diffusion problem with a random diffusion coefficient:

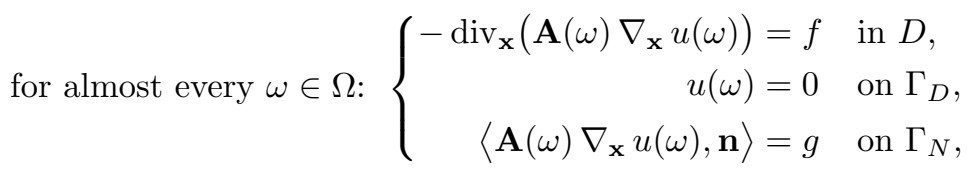


where $D \subset \mathbb{R}^{d}$ is a Lipschitz domain with $d \geq 1$ and $\partial D=\bar{\Gamma}_{D} \cup \bar{\Gamma}_{N}$ is a disjoint decomposition of the boundary. The function $f \in \widetilde{H}^{-1}(D)$ describes the known source and $g \in H^{-1 / 2}\left(\Gamma_{N}\right)$ the known conormal derivative at the Neumann boundary. The random matrix field $\mathbf{A} \in L_{\mathbb{P}}^{\infty}\left(\Omega ; L^{\infty}\left(D ; \mathbb{R}^{d \times d}\right)\right)$ is the stochastically and spatially varying diffusion coefficient, which satisfies the uniform ellipticity condition

$$
a_{\min } \leq \underset{\mathbf{x} \in D}{\operatorname{essinf}} \lambda_{\min }(\mathbf{A}(\mathbf{x}, \omega)) \leq \underset{\mathbf{x} \in D}{\operatorname{ess} \sup } \lambda_{\max }(\mathbf{A}(\mathbf{x}, \omega)) \leq a_{\max } \quad \mathbb{P} \text {-almost surely }
$$

for some constants $0<a_{\min } \leq a_{\max }<\infty$ and is almost surely symmetric almost everywhere. Without loss of generality, we assume $a_{\min } \leq 1$ and $a_{\max } \geq 1$.

To be able to capture the two situations arising from the fact that we might have a pure Neumann boundary value problem, we introduce the solution space $V$ dependent upon the boundary measure of $\Gamma_{D}$ : If $\Gamma_{D}$ has nonzero boundary measure, then we define

$$
V:=H_{\Gamma_{D}}^{1}(D):=\left\{v \in H^{1}(D): v(\mathbf{x})=0 \text { for all } \mathbf{x} \in \Gamma_{D}\right\} .
$$

If $\Gamma_{D}$ has zero boundary measure, i.e., if we have a pure Neumann boundary value problem, then we set

$$
V:=H_{*}^{1}(D):=\left\{v \in H^{1}(D):(v, 1)_{L^{2}(D)}=0\right\}
$$

and also require that $f$ and $g$ fulfil the compatibility condition

$$
\int_{D} f(\mathbf{x}) \mathrm{d} \mathbf{x}=-\int_{\Gamma_{N}} g(\mathbf{x}) \mathrm{d} s .
$$

In both cases, the norm equivalence theorem of Sobolev (see [2]), implies for all $v \in V$ and some constant $0<c_{V} \leq 1$ that

$$
c_{V}\|v\|_{H^{1}(D)} \leq\|v\|_{V}=\left\|\nabla_{\mathbf{x}} v\right\|_{L^{2}\left(D ; \mathbb{R}^{d}\right)} \leq\|v\|_{H^{1}(D)} .
$$

We will consider anisotropic diffusion coefficients that are of the form

$$
\mathbf{A}(\mathbf{x}, \omega):=a \mathbf{I}+\left(\|\mathbf{V}(\mathbf{x}, \omega)\|_{2}-a\right) \frac{\mathbf{V}(\mathbf{x}, \omega) \mathbf{V}^{\top}(\mathbf{x}, \omega)}{\mathbf{V}^{\top}(\mathbf{x}, \omega) \mathbf{V}(\mathbf{x}, \omega)},
$$

where $a \in \mathbb{R}$ is a given value and $\mathbf{V} \in L_{\mathbb{P}}^{\infty}\left(\Omega ; L^{\infty}\left(D ; \mathbb{R}^{d}\right)\right)$ is a random vector field. Furthermore, we require that they satisfy $b_{\min } \leq a \leq b_{\max }$ and

$$
b_{\min } \leq \underset{\mathbf{x} \in D}{\operatorname{essinf}}\|\mathbf{V}(\mathbf{x}, \omega)\|_{2} \leq \underset{\mathbf{x} \in D}{\operatorname{ess} \sup }\|\mathbf{V}(\mathbf{x}, \omega)\|_{2} \leq b_{\max } \quad \mathbb{P} \text {-almost surely }
$$

for some constants $0<b_{\min } \leq b_{\max }<\infty$. Without loss of generality, we assume $b_{\min } \leq 1$ and $b_{\max } \geq 1$.

We note that the field $\mathbf{A}$ accounts for a medium that has homogeneous diffusion strength $a$ perpendicular to $\mathbf{V}$ and has diffusion strength $\|\mathbf{V}(\mathbf{x}, \omega)\|_{2}$ in the direction of $\mathbf{V}$. The randomness of the specific direction and length of $\mathbf{V}$ therefore quantifies the uncertainty of this notable direction and its diffusion strength.

LEMma 2.1. A diffusion coefficient of form (3) is well-formed and indeed also satisfies the uniform ellipticity condition (2) with $b_{\min }$ and $b_{\max }$. 
Proof. For almost every $\omega \in \Omega$ and almost every $\mathbf{x} \in D$ we have that $\mathbf{A}(\mathbf{x}, \omega)$ is well-formed because of

$$
\mathbf{V}^{\top}(\mathbf{x}, \omega) \mathbf{V}(\mathbf{x}, \omega)=\|\mathbf{V}(\mathbf{x}, \omega)\|_{2}^{2} \geq b_{\min }^{2}>0,
$$

and clearly symmetric. Furthermore, we can choose $\mathbf{u}_{2}, \ldots, \mathbf{u}_{d} \in \mathbb{R}^{d}$ that are perpendicular to $\mathbf{V}(\mathbf{x}, \omega)$ and are linearly independent; then, we know that, for all $i=2, \ldots, d$,

$$
\mathbf{A}(\mathbf{x}, \omega) \mathbf{u}_{i}=a \mathbf{u}_{i} \quad \text { and } \quad \mathbf{A}(\mathbf{x}, \omega) \mathbf{V}(\mathbf{x}, \omega)=\|\mathbf{V}(\mathbf{x}, \omega)\|_{2} \mathbf{V}(\mathbf{x}, \omega) .
$$

This means that, for almost every $\omega \in \Omega$ and almost every $\mathbf{x} \in D$,

$$
\begin{aligned}
& \lambda_{\min }(\mathbf{A}(\mathbf{x}, \omega))=\min \left\{a,\|\mathbf{V}(\mathbf{x}, \omega)\|_{2}\right\} \geq b_{\min } \\
& \lambda_{\max }(\mathbf{A}(\mathbf{x}, \omega))=\max \left\{a,\|\mathbf{V}(\mathbf{x}, \omega)\|_{2}\right\} \leq b_{\max }
\end{aligned}
$$

Therefore, A satisfies the uniform ellipticity condition (2) with $b_{\min }$ and $b_{\max }$.

Thus, we will set $a_{\min }:=b_{\min }$ and $a_{\max }:=b_{\max }$ and, from here on, solely use $a_{\min }$ and $a_{\max }$.

\section{Problem reformulation.}

3.1. Karhunen-Loève expansion. To make the random field and, hence, also the diffusion coefficient, feasible for numerical computations, we separate the spatial variable $\mathbf{x}$ and the stochastic parameter $\omega$ by considering the Karhunen-Loève expansion of $\mathbf{V}$. The mean field $\mathbb{E}[\mathbf{V}]: \Omega \rightarrow \mathbb{R}^{d}$ and the matrix-valued covariance field $\operatorname{Cov}[\mathbf{V}]: D \times D \rightarrow \mathbb{R}^{d \times d}$ are given by

$$
\mathbb{E}[\mathbf{V}](\mathbf{x})=\int_{\Omega} \mathbf{V}(\mathbf{x}, \omega) \mathrm{d} \mathbb{P}(\omega)
$$

and

$$
\operatorname{Cov}[\mathbf{V}]\left(\mathbf{x}, \mathbf{x}^{\prime}\right)=\int_{\Omega} \mathbf{V}_{0}(\mathbf{x}, \omega) \mathbf{V}_{0}^{\top}\left(\mathbf{x}^{\prime}, \omega\right) \mathrm{d} \mathbb{P}(\omega),
$$

respectively, where

$$
\mathbf{V}_{0}(\mathbf{x}, \omega):=\mathbf{V}(\mathbf{x}, \omega)-\mathbb{E}[\mathbf{V}](\mathbf{x}) .
$$

Let $\left\{\lambda_{k}, \boldsymbol{\psi}_{k}\right\}_{k}$ denote the eigenpairs corresponding to the Hilbert-Schmidt operator $\mathcal{C}$ that is induced from the kernel $\operatorname{Cov}[\mathbf{V}]\left(\mathbf{x}, \mathbf{x}^{\prime}\right)$, i.e.,

$$
(\mathcal{C} \mathbf{u})(\mathbf{x}):=\int_{D} \operatorname{Cov}[\mathbf{V}]\left(\mathbf{x}, \mathbf{x}^{\prime}\right) \mathbf{u}\left(\mathbf{x}^{\prime}\right) \mathrm{d} \mathbf{x}^{\prime}
$$

Then, the Karhunen-Loève expansion of $\mathbf{V}$ is given by

$$
\mathbf{V}(\mathbf{x}, \omega)=\mathbb{E}[\mathbf{V}](\mathbf{x})+\sum_{k=1}^{\infty} \sqrt{\lambda_{k}} \boldsymbol{\psi}_{k}(\mathbf{x}) Y_{k}(\omega)
$$

where the uncorrelated and centered random variables $\left\{Y_{k}\right\}_{k}$ are given according to 


$$
Y_{k}(\omega):=\frac{1}{\sqrt{\lambda_{k}}} \int_{D} \mathbf{V}_{0}^{\top}(\mathbf{x}, \omega) \boldsymbol{\psi}_{k}(\mathbf{x}) \mathrm{d} \mathbf{x} .
$$

We note that the convergence of $(5)$ is naturally in the $L_{\mathbb{P}}^{2}\left(\Omega ; L^{2}\left(D ; \mathbb{R}^{d}\right)\right)$-norm. However, as shown in Lemma A.1, there holds $\operatorname{img}(\mathcal{C}) \subset L^{\infty}\left(D ; \mathbb{R}^{d}\right)$. This particularly implies that $\boldsymbol{\psi}_{k} \in L^{\infty}\left(D ; \mathbb{R}^{d}\right)$ and, as a consequence, also $Y_{k}(\omega) \in L_{\mathbb{P}}^{\infty}(\Omega)$.

Now, by parametrization of the $Y_{k}$ as $y_{k}$ and replacing the $\sqrt{\lambda_{k}}$ with $\sigma_{k}$, we may assume, without loss of generality, that $y_{k} \in[-1,1]$, when considering the vector field $\mathbf{V}$ in the parametrized form

$$
\mathbf{V}(\mathbf{x}, \mathbf{y})=\mathbb{E}[\mathbf{V}](\mathbf{x})+\sum_{k=1}^{\infty} \sigma_{k} \boldsymbol{\psi}_{k}(\mathbf{x}) y_{k}
$$

where $\mathbf{y}=\left(y_{k}\right)_{k \in \mathbb{N}} \in \square:=[-1,1]^{\mathbb{N}}$. Consequently, we can also view $\mathbf{A}(\mathbf{x}, \mathbf{y})$ and $u(\mathbf{x}, \mathbf{y})$ as being parametrized by $\mathbf{y}$ and restate (1) as

$$
\text { for almost every } \mathbf{y} \in \square:\left\{\begin{aligned}
-\operatorname{div}_{\mathbf{x}}\left(\mathbf{A}(\mathbf{y}) \nabla_{\mathbf{x}} u(\mathbf{y})\right)=f & \text { in } D, \\
u(\mathbf{y})=0 & \text { on } \Gamma_{D}, \\
\left\langle\mathbf{A}(\mathbf{y}) \nabla_{\mathbf{x}} u(\mathbf{y}), \mathbf{n}\right\rangle=g & \text { on } \Gamma_{N} .
\end{aligned}\right.
$$

We now impose some common assumptions, which make the Karhunen-Loève expansion computationally feasible.

Assumption 3.1. The random variables $\left(Y_{k}\right)_{k \in \mathbb{N}}$ are independent and uniformly distributed on $[-\sqrt{3}, \sqrt{3}]$, i.e., $\sigma_{k}=\sqrt{3 \lambda_{k}}$. Moreover, the sequence $\gamma=\left(\gamma_{k}\right)_{k \in \mathbb{N}_{0}}$, given by

$$
\gamma_{k}:=\left\|\sigma_{k} \boldsymbol{\psi}_{k}\right\|_{L^{\infty}\left(D ; \mathbb{R}^{d}\right)},
$$

is at least in $\ell^{1}\left(\mathbb{N}_{0}\right)$, where we have defined $\boldsymbol{\psi}_{0}:=\mathbb{E}[\mathbf{V}]$ and $\sigma_{0}:=1$.

The assumption guarantees that the representation (6) also converges in the Bochner space $L_{\mathbb{P}}^{\infty}\left(\Omega ; L^{\infty}\left(D ; \mathbb{R}^{d}\right)\right)$; see Lemma A.2 for a proof of this statement.

3.2. Spatially weak formulation. Since we want to pursue a finite element approach in space to approximate the solution of (7), we will need the spatially weak form thereof.

Given almost any $\mathbf{y} \in \square$, we have

$$
-\operatorname{div}_{\mathbf{x}}\left(\mathbf{A}(\mathbf{x}, \mathbf{y}) \nabla_{\mathbf{x}} u(\mathbf{x}, \mathbf{y})\right)=f(\mathbf{x}) \text { for all } \mathbf{x} \in D .
$$

After multiplication with a test function $v \in V$ and integration over $D$, we arrive at

$$
-\int_{D} \operatorname{div}_{\mathbf{x}}\left(\mathbf{A}(\mathbf{x}, \mathbf{y}) \nabla_{\mathbf{x}} u(\mathbf{x}, \mathbf{y})\right) v(\mathbf{x}) \mathrm{d} \mathbf{x}=\int_{D} f(\mathbf{x}) v(\mathbf{x}) \mathrm{d} \mathbf{x} .
$$

Now, Green's identity implies

$$
\begin{aligned}
-\int_{D} \operatorname{div}_{\mathbf{x}}\left(\mathbf{A}(\mathbf{x}, \mathbf{y}) \nabla_{\mathbf{x}} u(\mathbf{x}, \mathbf{y})\right) v(\mathbf{x}) \mathrm{d} \mathbf{x}= & \int_{D}\left\langle\mathbf{A}(\mathbf{x}, \mathbf{y}) \nabla_{\mathbf{x}} u(\mathbf{x}, \mathbf{y}), \nabla_{\mathbf{x}} v(\mathbf{x})\right\rangle \mathrm{d} \mathbf{x} \\
& -\int_{\partial D}\left\langle\mathbf{A}(\mathbf{x}, \mathbf{y}) \nabla_{\mathbf{x}} u(\mathbf{x}, \mathbf{y}), \mathbf{n}(\mathbf{x})\right\rangle v(\mathbf{x}) \mathrm{d} s,
\end{aligned}
$$

which, because of $\left.v\right|_{\Gamma_{D}}=0$ and $\left\langle\mathbf{A}(\mathbf{y}) \nabla_{\mathbf{x}} u(\mathbf{y}), \mathbf{n}\right\rangle=g$ on $\Gamma_{N}$, simplifies to 


$$
\begin{aligned}
-\int_{D} \operatorname{div}_{\mathbf{x}}\left(\mathbf{A}(\mathbf{x}, \mathbf{y}) \nabla_{\mathbf{x}} u(\mathbf{x}, \mathbf{y})\right) v(\mathbf{x}) \mathrm{d} \mathbf{x}= & \int_{D}\left\langle\mathbf{A}(\mathbf{x}, \mathbf{y}) \nabla_{\mathbf{x}} u(\mathbf{x}, \mathbf{y}), \nabla_{\mathbf{x}} v(\mathbf{x})\right\rangle \mathrm{d} \mathbf{x} \\
& -\int_{\Gamma_{N}} g(\mathbf{x}) v(\mathbf{x}) \mathrm{d} s .
\end{aligned}
$$

We define $\mathcal{B}: \square \rightarrow(V \times V \rightarrow \mathbb{R})$, where $\mathcal{B}[\mathbf{y}]$ is a continuous symmetric bilinear form for almost any $\mathbf{y} \in \square$, by

$$
\mathcal{B}[\mathbf{y}](u, v):=\int_{D}\left\langle\mathbf{A}(\mathbf{x}, \mathbf{y}) \nabla_{\mathbf{x}} u(\mathbf{x}), \nabla_{\mathbf{x}} v(\mathbf{x})\right\rangle \mathrm{d} \mathbf{x}
$$

and $\ell: V \rightarrow \mathbb{R}$ a continuous linear form by

$$
\ell(v):=\int_{D} f(\mathbf{x}) v(\mathbf{x}) \mathrm{d} \mathbf{x}+\int_{\Gamma_{N}} g(\mathbf{x}) v(\mathbf{x}) \mathrm{d} s .
$$

Then, this leads us to the spatially weak formulation

$$
\left\{\begin{array}{l}
\text { Find } u: \square \rightarrow V \text { such that } \\
\quad \mathcal{B}[\mathbf{y}](u(\mathbf{y}), v)=\ell(v) \quad \text { for almost every } \mathbf{y} \in \square \text { and all } v \in V .
\end{array}\right.
$$

We conclude with the following well-known stability estimate.

Lemma 3.2. For almost every $\mathbf{y} \in \square$, there is a unique solution $u(\mathbf{y}) \in V$ of (8), which fulfils

$$
\|u(\mathbf{y})\|_{H^{1}(D)} \leq \frac{a_{\max }}{a_{\min } c_{V}^{2}}\left(\|f\|_{\widetilde{H}^{-1}(D)}+\|g\|_{H^{-1 / 2}\left(\Gamma_{N}\right)}\right)
$$

\section{Parametric regularity.}

4.1. Parametric regularity of the diffusion coefficient. In this paragraph, we consider the parametric regularity of the diffusion coefficient. We will show that its derivatives exhibit a decay, which directly corresponds to the decay of the vector field's Karhunen-Loève expansion. In the following, we use the notation

$$
\begin{gathered}
\|s\|:=\|s\|_{L_{\mathbb{P}_{\mathbf{y}}}^{\infty}\left(\square ; L^{\infty}(D ; \mathbb{R})\right)}=\underset{\mathbf{y} \in \square}{\operatorname{ess} \sup } \underset{\mathbf{x} \in D}{\operatorname{essup}}|s(\mathbf{x}, \mathbf{y})|, \\
\|\mathbf{v}\|_{d}:=\|\mathbf{v}\|_{L_{\mathbb{P}_{\mathbf{y}}}^{\infty}\left(\square ; L^{\infty}\left(D ; \mathbb{R}^{d}\right)\right)}=\underset{\mathbf{y} \in \square}{\operatorname{ess} \sup } \underset{\mathbf{x} \in D}{\operatorname{ess} \sup }\|\mathbf{v}(\mathbf{x}, \mathbf{y})\|_{2}, \\
\|\mathbf{M}\|_{d \times d}:=\|\mathbf{M}\|_{L_{\mathbb{P}_{\mathbf{y}}}^{\infty}\left(\square ; L^{\infty}\left(D ; \mathbb{R}^{d \times d}\right)\right)}=\underset{\mathbf{y} \in \square}{\operatorname{ess} \sup \operatorname{ess} \sup }\|\mathbf{M}(\mathbf{x}, \mathbf{y})\|_{F}
\end{gathered}
$$

for $s \in L_{\mathbb{P}_{\mathbf{y}}}^{\infty}\left(\square ; L^{\infty}(D ; \mathbb{R})\right), \mathbf{v} \in L_{\mathbb{P}_{\mathbf{y}}}^{\infty}\left(\square ; L^{\infty}\left(D ; \mathbb{R}^{d}\right)\right)$, and $\mathbf{M} \in L_{\mathbb{P}_{\mathbf{y}}}^{\infty}\left(\square ; L^{\infty}\left(D ; \mathbb{R}^{d \times d}\right)\right)$. We will further make extensive use of the following straightforward result.

LemmA 4.1. Given $\mathbf{v}, \mathbf{w} \in L_{\mathbb{P}_{\mathbf{y}}}^{\infty}\left(\square ; L^{\infty}\left(D ; \mathbb{R}^{d}\right)\right)$, we have

$$
\left\|\mathbf{v}^{\top} \mathbf{w}\right\| \leq\|\mathbf{v}\|_{d}\|\mathbf{w}\|_{d} \quad \text { and } \quad\left\|\mathbf{v}^{\top} \mathbf{v}\right\|=\|\mathbf{v}\|_{d}^{2}
$$

as well as

$$
\left\|\mathbf{v} \mathbf{w}^{\top}\right\|_{d \times d} \leq\|\mathbf{v}\|_{d}\|\mathbf{w}\|_{d} \quad \text { and } \quad\left\|\mathbf{v v}^{\top}\right\|_{d \times d}=\|\mathbf{v}\|_{d}^{2}
$$


In this section, we assume that the vector field $\mathbf{V}$ is given by a finite rank Karhunen-Loève expansion, i.e.,

$$
\mathbf{V}(\mathbf{x}, \mathbf{y})=\boldsymbol{\psi}_{0}(\mathbf{x})+\sum_{k=1}^{M} \sigma_{k} \boldsymbol{\psi}_{k}(\mathbf{x}) y_{k}
$$

If necessary this can be attained by appropriate truncation; see Lemma A.3 for a proof of this statement.

We shall now provide regularity estimates for the different terms in (3).

Lemma 4.2. Let $\mathbf{B}$ be defined as $\mathbf{B}(\mathbf{x}, \mathbf{y}):=\mathbf{V}(\mathbf{x}, \mathbf{y}) \mathbf{V}^{\top}(\mathbf{x}, \mathbf{y})$. Then, we have for all $\boldsymbol{\alpha} \in \mathbb{N}_{0}^{M}$ that

$$
\left\|\partial_{\mathbf{y}}^{\boldsymbol{\alpha}} \mathbf{B}\right\|_{d \times d} \leq 2 a_{\max }^{2} \gamma^{\boldsymbol{\alpha}} .
$$

Proof. More verbosely, $\mathbf{B}$ is given by

$$
\mathbf{B}(\mathbf{x}, \mathbf{y})=\left(\boldsymbol{\psi}_{0}(\mathbf{x})+\sum_{k=1}^{M} \sigma_{k} \boldsymbol{\psi}_{k}(\mathbf{x}) y_{k}\right)\left(\boldsymbol{\psi}_{0}(\mathbf{x})+\sum_{k=1}^{M} \sigma_{k} \boldsymbol{\psi}_{k}(\mathbf{x}) y_{k}\right)^{\top},
$$

from which we can derive the first order derivatives, yielding

$$
\begin{aligned}
\partial_{y_{i}} \mathbf{B}(\mathbf{x}, \mathbf{y})= & \sigma_{i} \boldsymbol{\psi}_{i}(\mathbf{x})\left(\boldsymbol{\psi}_{0}(\mathbf{x})+\sum_{k=1}^{M} \sigma_{k} \boldsymbol{\psi}_{k}(\mathbf{x}) y_{k}\right)^{\top} \\
& +\left(\boldsymbol{\psi}_{0}(\mathbf{x})+\sum_{k=1}^{M} \sigma_{k} \boldsymbol{\psi}_{k}(\mathbf{x}) y_{k}\right) \sigma_{i} \boldsymbol{\psi}_{i}^{\top}(\mathbf{x}),
\end{aligned}
$$

and from those also the second order derivatives. They are given by

$$
\partial_{y_{j}} \partial_{y_{i}} \mathbf{B}(\mathbf{x}, \mathbf{y})=\sigma_{i} \boldsymbol{\psi}_{i}(\mathbf{x}) \sigma_{j} \boldsymbol{\psi}_{j}^{\top}(\mathbf{x})+\sigma_{j} \boldsymbol{\psi}_{j}(\mathbf{x}) \sigma_{i} \boldsymbol{\psi}_{i}^{\top}(\mathbf{x})
$$

Since the second order derivatives with respect to $\mathbf{y}$ are constant, all higher order derivatives with respect to $\mathbf{y}$ vanish. bound

We obviously have $\|\mathbf{B}\|_{d \times d}=\|\mathbf{V}\|_{d}^{2} \leq a_{\max }^{2}$. From (9) we can now derive the

$$
\left\|\partial_{y_{i}} \mathbf{B}\right\|_{d \times d} \leq 2\left\|\sigma_{i} \boldsymbol{\psi}_{i}\right\|_{d}\left\|\boldsymbol{\psi}_{0}+\sum_{k=1}^{M} \sigma_{k} \boldsymbol{\psi}_{k} y_{k}\right\|_{d} \leq 2 \gamma_{i} a_{\max }
$$

and (10) leads us to $\left\|\partial_{y_{j}} \partial_{y_{i}} \mathbf{B}\right\|_{d \times d} \leq 2\left\|\sigma_{i} \boldsymbol{\psi}_{i}\right\|_{d}\left\|\sigma_{j} \boldsymbol{\psi}_{j}\right\|_{d} \leq 2 \gamma_{i} \gamma_{j}$. Therefore, we have

$$
\left\|\partial_{\mathbf{y}}^{\boldsymbol{\alpha}} \mathbf{B}\right\|_{d \times d} \leq \begin{cases}a_{\max }^{2} \gamma^{\boldsymbol{\alpha}} & \text { if }|\boldsymbol{\alpha}|=0, \\ 2 a_{\max } \gamma^{\boldsymbol{\alpha}} & \text { if }|\boldsymbol{\alpha}|=1, \\ 2 \boldsymbol{\gamma}^{\boldsymbol{\alpha}} & \text { if }|\boldsymbol{\alpha}|=2, \\ 0 & \text { if }|\boldsymbol{\alpha}|>2,\end{cases}
$$

and are finished since $a_{\max } \geq 1$.

Lemma 4.3. Let us define $C(\mathbf{x}, \mathbf{y}):=\mathbf{V}^{\top}(\mathbf{x}, \mathbf{y}) \mathbf{V}(\mathbf{x}, \mathbf{y}), D(\mathbf{x}, \mathbf{y}):=(C(\mathbf{x}, \mathbf{y}))^{-1}$, and $E(\mathbf{x}, \mathbf{y}):=\sqrt{C(\mathbf{x}, \mathbf{y})}$. Then, we know for all $\boldsymbol{\alpha} \in \mathbb{N}_{0}^{M}$ that

$\left\|\partial_{\mathbf{y}}^{\boldsymbol{\alpha}} D\right\| \leq|\boldsymbol{\alpha}| ! \frac{1}{a_{\min }^{2}}\left(\frac{2 a_{\max }^{2}}{a_{\min }^{2} \log 2}\right)^{|\boldsymbol{\alpha}|} \boldsymbol{\gamma}^{\boldsymbol{\alpha}} \quad$ and $\quad\left\|\partial_{\mathbf{y}}^{\boldsymbol{\alpha}} E\right\| \leq|\boldsymbol{\alpha}| ! a_{\max }\left(\frac{2 a_{\max }^{2}}{a_{\min }^{2} \log 2}\right)^{|\boldsymbol{\alpha}|} \boldsymbol{\gamma}^{\boldsymbol{\alpha}}$.

Copyright $\odot$ by SIAM. Unauthorized reproduction of this article is prohibited. 
Proof. The function $C$ can be expressed as

$$
C(\mathbf{x}, \mathbf{y})=\left(\boldsymbol{\psi}_{0}(\mathbf{x})+\sum_{k=1}^{M} \sigma_{k} \boldsymbol{\psi}_{k}(\mathbf{x}) y_{k}\right)^{\top}\left(\boldsymbol{\psi}_{0}(\mathbf{x})+\sum_{k=1}^{M} \sigma_{k} \boldsymbol{\psi}_{k}(\mathbf{x}) y_{k}\right),
$$

which, by derivation, gives the following expressions for the first order derivatives,

$$
\begin{aligned}
\partial_{y_{i}} C(\mathbf{x}, \mathbf{y})= & \sigma_{i} \boldsymbol{\psi}_{i}^{\top}(\mathbf{x})\left(\boldsymbol{\psi}_{0}(\mathbf{x})+\sum_{k=1}^{M} \sigma_{k} \boldsymbol{\psi}_{k}(\mathbf{x}) y_{k}\right) \\
& +\left(\boldsymbol{\psi}_{0}(\mathbf{x})+\sum_{k=1}^{M} \sigma_{k} \boldsymbol{\psi}_{k}(\mathbf{x}) y_{k}\right)^{\top} \sigma_{i} \boldsymbol{\psi}_{i}(\mathbf{x})
\end{aligned}
$$

Computing the second order derivatives then yields

$$
\partial_{y_{j}} \partial_{y_{i}} C(\mathbf{x}, \mathbf{y})=\sigma_{i} \boldsymbol{\psi}_{i}^{\top}(\mathbf{x}) \sigma_{j} \boldsymbol{\psi}_{j}(\mathbf{x})+\sigma_{j} \boldsymbol{\psi}_{j}^{\top}(\mathbf{x}) \sigma_{i} \boldsymbol{\psi}_{i}(\mathbf{x})
$$

and all higher order derivatives with respect to $\mathbf{y}$ are zero, since the second order derivatives with respect to $\mathbf{y}$ are already constant.

We use (4) to arrive at $a_{\min }^{2} \leq\|C\|=\|\mathbf{V}\|_{d}^{2} \leq a_{\max }^{2}$, which also yields

$$
\frac{1}{a_{\max }^{2}} \leq\|D\| \leq \frac{1}{a_{\min }^{2}} \quad \text { and } \quad a_{\min } \leq\|E\| \leq a_{\max }
$$

Using (11) yields the bound

$$
\left\|\partial_{y_{i}} C\right\| \leq 2\left\|\sigma_{i} \boldsymbol{\psi}_{i}\right\|_{d}\left\|\boldsymbol{\psi}_{0}+\sum_{k=1}^{M} \sigma_{k} \boldsymbol{\psi}_{k} y_{k}\right\|_{d} \leq 2 \gamma_{i} a_{\max }
$$

and, from (12), we can derive the bound $\left\|\partial_{y_{j}} \partial_{y_{i}} C\right\| \leq 2\left\|\sigma_{i} \boldsymbol{\psi}_{i}\right\|\left\|_{d}\right\| \sigma_{j} \boldsymbol{\psi}_{j} \|_{d} \leq 2 \gamma_{i} \gamma_{j}$. Thus, we know that

$$
\left\|\partial_{\mathbf{y}}^{\boldsymbol{\alpha}} C\right\| \leq 2 a_{\max }^{2} \gamma^{\boldsymbol{\alpha}} .
$$

Because $D=v \circ C$ with $v(x)=x^{-1}$ and $E=w \circ C$ with $w(x)=\sqrt{x}$ are composite functions, we employ the Faà di Bruno formula (see [7]), to compute their derivatives. The $r$ th derivative of $v$ is given by

$$
\frac{\mathrm{d}^{r}}{\mathrm{~d} x^{r}} v(x)=(-1)^{r} r ! x^{-1-r}=(-1)^{r} r ! v(x)^{r+1}
$$

and the $r$ th derivative of $w$ is given by

$$
\frac{\mathrm{d}^{r}}{\mathrm{~d} x^{r}} w(x)=c_{r} x^{\frac{1}{2}-r}=c_{r} w(x) v(x)^{r},
$$

where $c_{r}:=\prod_{i=0}^{r-1}\left(\frac{1}{2}-i\right)$. For $n=|\boldsymbol{\alpha}|$ we thus arrive at

$$
\partial_{\mathbf{y}}^{\boldsymbol{\alpha}} D(\mathbf{x}, \mathbf{y})=\sum_{r=1}^{n}(-1)^{r} r ! D(\mathbf{x}, \mathbf{y})^{r+1} \sum_{P(\boldsymbol{\alpha}, r)} \boldsymbol{\alpha} ! \prod_{j=1}^{n} \frac{\left(\partial_{\mathbf{y}}^{\boldsymbol{\beta}_{j}} C(\mathbf{x}, \mathbf{y})\right)^{k_{j}}}{k_{j} !\left(\boldsymbol{\beta}_{j} !\right)^{k_{j}}}
$$

Copyright $@$ by SIAM. Unauthorized reproduction of this article is prohibited. 
and

$$
\partial_{\mathbf{y}}^{\boldsymbol{\alpha}} E(\mathbf{x}, \mathbf{y})=\sum_{r=1}^{n} c_{r} E(\mathbf{x}, \mathbf{y}) D(\mathbf{x}, \mathbf{y})^{r} \sum_{P(\boldsymbol{\alpha}, r)} \boldsymbol{\alpha} ! \prod_{j=1}^{n} \frac{\left(\partial_{\mathbf{y}}^{\boldsymbol{\beta}_{j}} C(\mathbf{x}, \mathbf{y})\right)^{k_{j}}}{k_{j} !\left(\boldsymbol{\beta}_{j} !\right)^{k_{j}}}
$$

where $P(\boldsymbol{\alpha}, r)$ is a subset of integer partitions of a multiindex $\boldsymbol{\alpha}$ into $r$ nonvanishing multi-indices, given by

$$
P(\boldsymbol{\alpha}, r):=\left\{\left(\left(k_{1}, \boldsymbol{\beta}_{1}\right), \ldots,\left(k_{n}, \boldsymbol{\beta}_{n}\right)\right) \in\left(\mathbb{N}_{0} \times \mathbb{N}_{0}^{M}\right)^{n}: \sum_{j=1}^{n} k_{j} \boldsymbol{\beta}_{j}=\boldsymbol{\alpha}, \sum_{j=1}^{n} k_{i}=r\right.
$$

and there exists $1 \leq s \leq n: k_{j}=0$ and $\boldsymbol{\beta}_{j}=\mathbf{0}$ for all $1 \leq j \leq n-s$,

$$
\left.k_{j}>0 \text { for all } n-s+1 \leq j \leq n \text { and } \mathbf{0} \prec \boldsymbol{\beta}_{n-s+1} \prec \cdots \boldsymbol{\beta}_{n}\right\} \text {. }
$$

The relation $\boldsymbol{\beta} \prec \boldsymbol{\beta}^{\prime}$ for multi-indices $\boldsymbol{\beta}, \boldsymbol{\beta}^{\prime} \in \mathbb{N}_{0}^{M}$ means that either $|\boldsymbol{\beta}|<\left|\boldsymbol{\beta}^{\prime}\right|$ or, when $|\boldsymbol{\beta}|=\left|\boldsymbol{\beta}^{\prime}\right|$, there exists $0 \leq k<m$ such that $\beta_{1}=\beta_{1}^{\prime}, \ldots, \beta_{k}=\beta_{k}^{\prime}$ and $\beta_{k+1}<\beta_{k+1}^{\prime}$.

Taking the norm of (13) and (14) leads us to

$$
\begin{aligned}
\left\|\partial_{\mathbf{y}}^{\boldsymbol{\alpha}} D\right\| & \leq \sum_{r=1}^{n} r !\|D\|^{r+1} \sum_{P(\boldsymbol{\alpha}, r)} \boldsymbol{\alpha} ! \prod_{j=1}^{n} \frac{\left\|\partial_{\mathbf{y}}^{\boldsymbol{\beta}_{j}} C\right\|^{k_{j}}}{k_{j} !\left(\boldsymbol{\beta}_{j} !\right)^{k_{j}}} \\
& \leq \sum_{r=1}^{n} r !\left(\frac{1}{a_{\min }^{2}}\right)^{r+1} \sum_{P(\boldsymbol{\alpha}, r)} \boldsymbol{\alpha} ! \prod_{j=1}^{n} \frac{\left(2 a_{\max }^{2} \boldsymbol{\gamma}^{\left.\boldsymbol{\beta}_{j}\right)^{k_{j}}}\right.}{k_{j} !\left(\boldsymbol{\beta}_{j} !\right)^{k_{j}}} \\
& =\boldsymbol{\gamma}^{\boldsymbol{\alpha}} \sum_{r=1}^{n} r !\left(\frac{1}{a_{\min }^{2}}\right)^{r+1}\left(2 a_{\max }^{2}\right)^{r} \sum_{P(\boldsymbol{\alpha}, r)} \boldsymbol{\alpha} ! \prod_{j=1}^{n} \frac{1}{k_{j} !\left(\boldsymbol{\beta}_{j} !\right)^{k_{j}}}
\end{aligned}
$$

and

$$
\begin{aligned}
\left\|\partial_{\mathbf{y}}^{\boldsymbol{\alpha}} E\right\| \mid & \leq \sum_{r=1}^{n}\left|c_{r}\right|\left\|\left|E\|\mid\| D \|^{r} \sum_{P(\boldsymbol{\alpha}, r)} \boldsymbol{\alpha} ! \prod_{j=1}^{n} \frac{\left\|\partial_{\mathbf{y}}^{\boldsymbol{\beta}_{j}} C\right\|^{k_{j}}}{k_{j} !\left(\boldsymbol{\beta}_{j} !\right)^{k_{j}}}\right.\right. \\
& \leq \sum_{r=1}^{n}\left|c_{r}\right| a_{\max }\left(\frac{1}{a_{\min }^{2}}\right)^{r} \sum_{P(\boldsymbol{\alpha}, r)} \boldsymbol{\alpha} ! \prod_{j=1}^{n} \frac{\left(2 a_{\max }^{2} \boldsymbol{\gamma}^{\boldsymbol{\beta}_{j}}\right)^{k_{j}}}{k_{j} !\left(\boldsymbol{\beta}_{j} !\right)^{k_{j}}} \\
& =\boldsymbol{\gamma}^{\boldsymbol{\alpha}} \sum_{r=1}^{n}\left|c_{r}\right| a_{\max }\left(\frac{1}{a_{\min }^{2}}\right)^{r}\left(2 a_{\max }^{2}\right)^{r} \sum_{P(\boldsymbol{\alpha}, r)} \boldsymbol{\alpha} ! \prod_{j=1}^{n} \frac{1}{k_{j} !\left(\boldsymbol{\beta}_{j} !\right)^{k_{j}}} .
\end{aligned}
$$

Since we know from [7] that

$$
\sum_{P(\boldsymbol{\alpha}, r)} \boldsymbol{\alpha} ! \prod_{j=1}^{n} \frac{1}{k_{j} !\left(\boldsymbol{\beta}_{j} !\right)^{k_{j}}}=S_{n, r}
$$

where $S_{n, r}$ denotes the Stirling numbers of the second kind (see [1]), and that $\left|c_{r}\right| \leq r$ !, we can obtain

$$
\left\|\partial_{\mathbf{y}}^{\boldsymbol{\alpha}} D\right\| \leq \frac{1}{a_{\min }^{2}} \gamma^{\boldsymbol{\alpha}} \sum_{r=1}^{n} r !\left(\frac{2 a_{\max }^{2}}{a_{\min }^{2}}\right)^{r} S_{n, r} \leq \frac{1}{a_{\min }^{2}}\left(\frac{2 a_{\max }^{2}}{a_{\min }^{2}}\right)^{|\boldsymbol{\alpha}|} \gamma^{\boldsymbol{\alpha}} \sum_{r=1}^{n} r ! S_{n, r}
$$

Copyright (C) by SIAM. Unauthorized reproduction of this article is prohibited. 
and

$$
\left\|\partial_{\mathbf{y}}^{\alpha} E\right\| \leq a_{\max } \gamma^{\alpha} \sum_{r=1}^{n} r !\left(\frac{2 a_{\max }^{2}}{a_{\min }^{2}}\right)^{r} S_{n, r} \leq a_{\max }\left(\frac{2 a_{\max }^{2}}{a_{\min }^{2}}\right)^{|\alpha|} \gamma^{\alpha} \sum_{r=1}^{n} r ! S_{n, r} .
$$

Because $\sum_{r=1}^{n} r ! S_{n, r}$ equals the $n$th ordered Bell number, we can bound it (see [5]), by

$$
\sum_{r=1}^{n} r ! S_{n, r} \leq \frac{n !}{(\log 2)^{n}}
$$

This implies the assertion.

By combining the previous two lemmas, we derive the following result.

Lemma 4.4. We define $\mathbf{F}$ by

$$
\mathbf{F}(\mathbf{x}, \mathbf{y}):=\frac{\mathbf{V}(\mathbf{x}, \mathbf{y}) \mathbf{V}^{\top}(\mathbf{x}, \mathbf{y})}{\mathbf{V}^{\top}(\mathbf{x}, \mathbf{y}) \mathbf{V}(\mathbf{x}, \mathbf{y})}
$$

Then, we have for all $\boldsymbol{\alpha} \in \mathbb{N}_{0}^{M}$ that

$$
\left\|\partial_{\mathbf{y}}^{\alpha} \mathbf{F}\right\|_{d \times d} \leq|\boldsymbol{\alpha}| ! \frac{6 a_{\max }^{2}}{a_{\min }^{2}}\left(\frac{2 a_{\max }^{2}}{a_{\min }^{2} \log 2}\right)^{|\boldsymbol{\alpha}|} \gamma^{\alpha} .
$$

Proof. We can equivalently state $\mathbf{F}$ as $\mathbf{F}(\mathbf{x}, \mathbf{y})=D(\mathbf{x}, \mathbf{y}) \mathbf{B}(\mathbf{x}, \mathbf{y})$. Then, by applying the Leibniz rule, we arrive at

$$
\partial_{\mathbf{y}}^{\boldsymbol{\alpha}} \mathbf{F}(\mathbf{x}, \mathbf{y})=\sum_{\boldsymbol{\beta} \leq \boldsymbol{\alpha}}\left(\begin{array}{c}
\boldsymbol{\alpha} \\
\boldsymbol{\beta}
\end{array}\right)\left(\partial_{\mathbf{y}}^{\boldsymbol{\beta}} D(\mathbf{x}, \mathbf{y})\right)\left(\partial_{\mathbf{y}}^{\boldsymbol{\alpha}-\boldsymbol{\beta}} \mathbf{B}(\mathbf{x}, \mathbf{y})\right) .
$$

Taking the norm and using the bounds from Lemmas 4.2 and 4.3 lead us to

$$
\begin{aligned}
\left\|\partial_{\mathbf{y}}^{\boldsymbol{\alpha}} \mathbf{F}\right\|_{d \times d} & \leq \sum_{\boldsymbol{\beta} \leq \boldsymbol{\alpha}}\left(\begin{array}{c}
\boldsymbol{\alpha} \\
\boldsymbol{\beta}
\end{array}\right)\left\|\partial_{\mathbf{y}}^{\boldsymbol{\beta}} D\right\|\left\|\partial_{\mathbf{y}}^{\boldsymbol{\alpha}-\boldsymbol{\beta}} \mathbf{B}\right\|_{d \times d} \\
& \leq \sum_{\boldsymbol{\beta} \leq \boldsymbol{\alpha}}\left(\begin{array}{c}
\boldsymbol{\alpha} \\
\boldsymbol{\beta}
\end{array}\right)|\boldsymbol{\beta}| ! \frac{1}{a_{\min }^{2}}\left(\frac{2 a_{\max }^{2}}{a_{\min }^{2} \log 2}\right)^{|\boldsymbol{\beta}|} \boldsymbol{\gamma}^{\boldsymbol{\beta}} 2 a_{\max }^{2} \boldsymbol{\gamma}^{\boldsymbol{\alpha}-\boldsymbol{\beta}} \\
& \leq \frac{2 a_{\max }^{2}}{a_{\min }^{2}}\left(\frac{2 a_{\max }^{2}}{a_{\min }^{2} \log 2}\right)^{|\boldsymbol{\alpha}|} \boldsymbol{\gamma}^{\boldsymbol{\alpha}} \sum_{\boldsymbol{\beta} \leq \boldsymbol{\alpha}}\left(\begin{array}{c}
\boldsymbol{\alpha} \\
\boldsymbol{\beta}
\end{array}\right)|\boldsymbol{\beta}| !
\end{aligned}
$$

Last, the combinatorial identity

$$
\sum_{\substack{\boldsymbol{\beta} \leq \boldsymbol{\alpha} \\
|\boldsymbol{\beta}|=j}}\left(\begin{array}{c}
\boldsymbol{\alpha} \\
\boldsymbol{\beta}
\end{array}\right)=\left(\begin{array}{c}
|\boldsymbol{\alpha}| \\
j
\end{array}\right)
$$

yields the bound

$$
\sum_{\boldsymbol{\beta} \leq \boldsymbol{\alpha}}\left(\begin{array}{c}
\boldsymbol{\alpha} \\
\boldsymbol{\beta}
\end{array}\right)|\boldsymbol{\beta}| !=\sum_{j=0}^{|\boldsymbol{\alpha}|} j ! \sum_{\substack{\boldsymbol{\beta} \leq \boldsymbol{\alpha} \\
|\boldsymbol{\beta}|=j}}\left(\begin{array}{c}
\boldsymbol{\alpha} \\
\boldsymbol{\beta}
\end{array}\right)=\sum_{j=0}^{|\boldsymbol{\alpha}|} j !\left(\begin{array}{c}
|\boldsymbol{\alpha}| \\
j
\end{array}\right)=|\boldsymbol{\alpha}| ! \sum_{k=0}^{|\boldsymbol{\alpha}|} \frac{1}{k !} \leq 3|\boldsymbol{\alpha}| ! .
$$

Copyright (c) by SIAM. Unauthorized reproduction of this article is prohibited. 
Gathering all the regularity estimates for the different terms in (3) gives us the regularity of the diffusion matrix $\mathbf{A}$.

TheOrem 4.5. The derivatives of the diffusion matrix $\mathbf{A}$ defined in (3) satisfy

$$
\left\|\partial_{\mathbf{y}}^{\boldsymbol{\alpha}} \mathbf{A}\right\|_{d \times d} \leq(|\boldsymbol{\alpha}|+1) !\left(2 a_{\max }\right) \frac{6 a_{\max }^{2}}{a_{\min }^{2}}\left(\frac{2 a_{\max }^{2}}{a_{\min }^{2} \log 2}\right)^{|\boldsymbol{\alpha}|} \gamma^{\boldsymbol{\alpha}}
$$

for all $\boldsymbol{\alpha} \in \mathbb{N}_{0}^{M}$ with $|\boldsymbol{\alpha}| \geq 1$.

Proof. We can state $\mathbf{A}$ as $\mathbf{A}(\mathbf{x}, \mathbf{y})=a \mathbf{I}+E(\mathbf{x}, \mathbf{y}) \mathbf{F}(\mathbf{x}, \mathbf{y})-a \mathbf{F}(\mathbf{x}, \mathbf{y})$, which, with the Leibniz rule, yields

$$
\partial_{\mathbf{y}}^{\boldsymbol{\alpha}} \mathbf{A}(\mathbf{x}, \mathbf{y})=\sum_{\boldsymbol{\beta} \leq \boldsymbol{\alpha}}\left(\begin{array}{c}
\boldsymbol{\alpha} \\
\boldsymbol{\beta}
\end{array}\right)\left(\partial_{\mathbf{y}}^{\boldsymbol{\beta}} E(\mathbf{x}, \mathbf{y})\right)\left(\partial_{\mathbf{y}}^{\boldsymbol{\alpha}-\boldsymbol{\beta}} \mathbf{F}(\mathbf{x}, \mathbf{y})\right)-a \partial_{\mathbf{y}}^{\boldsymbol{\alpha}} \mathbf{F}(\mathbf{x}, \mathbf{y}) .
$$

Then, by taking the norm and inserting the bounds from Lemmas 4.3 and 4.4 , we arrive at

$$
\begin{aligned}
\left\|\partial_{\mathbf{y}}^{\boldsymbol{\alpha}} \mathbf{A}\right\|_{d \times d} \leq & \sum_{\boldsymbol{\beta} \leq \boldsymbol{\alpha}}\left(\begin{array}{c}
\boldsymbol{\alpha} \\
\boldsymbol{\beta}
\end{array}\right)\left\|\partial_{\mathbf{y}}^{\boldsymbol{\beta}} E\right\|\left\|\partial_{\mathbf{y}}^{\boldsymbol{\alpha}-\boldsymbol{\beta}} \mathbf{F}\right\|_{d \times d}+a_{\max }\left\|\partial_{\mathbf{y}}^{\boldsymbol{\alpha}} \mathbf{F}\right\|_{d \times d} \\
\leq & \sum_{\boldsymbol{\beta} \leq \boldsymbol{\alpha}}\left(\begin{array}{c}
\boldsymbol{\alpha} \\
\boldsymbol{\beta}
\end{array}\right)|\boldsymbol{\beta}| ! a_{\max }\left(\frac{2 a_{\max }^{2}}{a_{\min }^{2} \log 2}\right)^{|\boldsymbol{\beta}|} \boldsymbol{\gamma}^{\boldsymbol{\beta}} \\
& \times|\boldsymbol{\alpha}-\boldsymbol{\beta}| ! \frac{6 a_{\max }^{2}}{a_{\min }^{2}}\left(\frac{2 a_{\max }^{2}}{a_{\min }^{2} \log 2}\right)^{|\boldsymbol{\alpha}-\boldsymbol{\beta}|} \boldsymbol{\gamma}^{\boldsymbol{\alpha}-\boldsymbol{\beta}} \\
& +|\boldsymbol{\alpha}| ! a_{\max } \frac{6 a_{\max }^{2}}{a_{\min }^{2}}\left(\frac{2 a_{\max }^{2}}{a_{\min }^{2} \log 2}\right)^{|\boldsymbol{\alpha}|} \boldsymbol{\gamma}^{\boldsymbol{\alpha}} \\
\leq & a_{\max } \frac{6 a_{\max }^{2}}{a_{\min }^{2}}\left(\frac{2 a_{\max }^{2}}{a_{\min }^{2} \log 2}\right)^{|\boldsymbol{\alpha}|} \boldsymbol{\gamma}^{\boldsymbol{\alpha}} \sum_{\boldsymbol{\beta} \leq \boldsymbol{\alpha}}\left(\begin{array}{c}
\boldsymbol{\alpha} \\
\boldsymbol{\beta}
\end{array}\right)|\boldsymbol{\beta}| !|\boldsymbol{\alpha}-\boldsymbol{\beta}| ! \\
& +a_{\max } \frac{6 a_{\max }^{2}}{a_{\text {min }}^{2}}\left(\frac{2 a_{\max }^{2}}{a_{\min }^{2} \log 2}\right)^{|\boldsymbol{\alpha}|} \boldsymbol{\gamma}^{\boldsymbol{\alpha}}|\boldsymbol{\alpha}| ! .
\end{aligned}
$$

Finally, the combinatorial identity (15) yields (see, e.g., [12]),

$$
\sum_{\boldsymbol{\beta} \leq \boldsymbol{\alpha}}\left(\begin{array}{c}
\boldsymbol{\alpha} \\
\boldsymbol{\beta}
\end{array}\right)|\boldsymbol{\beta}| !|\boldsymbol{\alpha}-\boldsymbol{\beta}| !=(|\boldsymbol{\alpha}|+1) !
$$

If we now define the modified sequence $\boldsymbol{\mu}=\left(\mu_{k}\right)_{k \in \mathbb{N}_{0}}$ as

$$
\mu_{k}:=\frac{4 a_{\max }^{2}}{a_{\min }^{2} \log 2} \gamma_{k} \quad \text { and also } \quad c_{\mathbf{A}}:=\left(2 a_{\max }\right) \frac{6 a_{\max }^{2}}{a_{\min }^{2}},
$$

we can summarize the results attained so far $b^{2}$

$$
\left\|\partial_{\mathbf{y}}^{\alpha} \mathbf{A}\right\|_{d \times d} \leq|\boldsymbol{\alpha}| ! c_{\mathbf{A}} \boldsymbol{\mu}^{\alpha}
$$

\footnotetext{
${ }^{2}$ Note that the additional factor of 2 in $\mu_{k}$ removes the factor $|\boldsymbol{\alpha}|+1$ from the factorial expression, since we know that $2^{|\boldsymbol{\alpha}|} \geq|\boldsymbol{\alpha}|+1$.
}

Copyright $($ C by SIAM. Unauthorized reproduction of this article is prohibited. 
4.2. Parametric regularity of the solution. Next, we show that the decay rates of the diffusion coefficient's derivatives carry over to the solution's derivatives.

THEOREM 4.6. For almost every $\mathbf{y} \in \square$, the derivatives of the solution $u(\mathbf{y})$ of (8) satisfy

$$
\left\|\partial_{\mathbf{y}}^{\boldsymbol{\alpha}} u(\mathbf{y})\right\|_{H^{1}(D)} \leq|\boldsymbol{\alpha}| ! \boldsymbol{\mu}^{\boldsymbol{\alpha}}\left(\frac{a_{\max }}{a_{\min } c_{V}^{2}} \max \left\{2 c_{\mathbf{A}},\|f\|_{\widetilde{H}^{-1}(D)}+\|g\|_{H^{-1 / 2}\left(\Gamma_{N}\right)}\right\}\right)^{|\boldsymbol{\alpha}|+1}
$$

Proof. By differentiation of the variational formulation (8) with respect to $\mathbf{y}$ we arrive, for arbitrary $v \in V$, at

$$
\left(\partial_{\mathbf{y}}^{\boldsymbol{\alpha}}\left(\mathbf{A}(\mathbf{y}) \nabla_{\mathbf{x}} u(\mathbf{y})\right), \nabla_{\mathbf{x}} v\right)_{L^{2}\left(D ; \mathbb{R}^{d}\right)}=0 .
$$

Applying the Leibniz rule on the left-hand side yields

$$
\left(\sum_{\boldsymbol{\beta} \leq \boldsymbol{\alpha}}\left(\begin{array}{c}
\boldsymbol{\alpha} \\
\boldsymbol{\beta}
\end{array}\right) \partial_{\mathbf{y}}^{\boldsymbol{\alpha}-\boldsymbol{\beta}} \mathbf{A}(\mathbf{y}) \partial_{\mathbf{y}}^{\boldsymbol{\beta}} \nabla_{\mathbf{x}} u(\mathbf{y}), \nabla_{\mathbf{x}} v\right)_{L^{2}\left(D ; \mathbb{R}^{d}\right)}=0 .
$$

Then, by rearranging and using the linearity of the gradient, we find

$$
\int_{D}\left\langle\mathbf{A}(\mathbf{y}) \nabla_{\mathbf{x}} \partial_{\mathbf{y}}^{\boldsymbol{\alpha}} u(\mathbf{y}), \nabla_{\mathbf{x}} v\right\rangle \mathrm{d} \mathbf{x}=-\sum_{\boldsymbol{\beta}<\boldsymbol{\alpha}}\left(\begin{array}{c}
\boldsymbol{\alpha} \\
\boldsymbol{\beta}
\end{array}\right) \int_{D}\left\langle\partial_{\mathbf{y}}^{\boldsymbol{\alpha}-\boldsymbol{\beta}} \mathbf{A}(\mathbf{y}) \nabla_{\mathbf{x}} \partial_{\mathbf{y}}^{\boldsymbol{\beta}} u(\mathbf{y}), \nabla_{\mathbf{x}} v\right\rangle \mathrm{d} \mathbf{x}
$$

We now choose $v=\partial_{\mathbf{y}}^{\mathbf{\alpha}} u(\mathbf{y})$ and employ the coercivity as well as the bound from Theorem 4.5. This results in

$$
\begin{aligned}
a_{\min } c_{V}^{2}\left\|\partial_{\mathbf{y}}^{\boldsymbol{\alpha}} u(\mathbf{y})\right\|_{H^{1}(D)}^{2} & \leq-\sum_{\boldsymbol{\beta}<\boldsymbol{\alpha}}\left(\begin{array}{c}
\boldsymbol{\alpha} \\
\boldsymbol{\beta}
\end{array}\right) \int_{D}\left\langle\partial_{\mathbf{y}}^{\boldsymbol{\alpha}-\boldsymbol{\beta}} \mathbf{A}(\mathbf{y}) \nabla_{\mathbf{x}} \partial_{\mathbf{y}}^{\boldsymbol{\beta}} u(\mathbf{y}), \nabla_{\mathbf{x}} \partial_{\mathbf{y}}^{\boldsymbol{\alpha}} u(\mathbf{y})\right\rangle \mathrm{d} \mathbf{x} \\
& \leq \sum_{\boldsymbol{\beta}<\boldsymbol{\alpha}}\left(\begin{array}{c}
\boldsymbol{\alpha} \\
\boldsymbol{\beta}
\end{array}\right)\left\|\partial_{\mathbf{y}}^{\boldsymbol{\alpha}-\boldsymbol{\beta}} \mathbf{A}\right\|\left\|_{d \times d}\right\| \partial_{\mathbf{y}}^{\boldsymbol{\beta}} u(\mathbf{y})\left\|_{H^{1}(D)}\right\| \partial_{\mathbf{y}}^{\boldsymbol{\alpha}} u(\mathbf{y}) \|_{H^{1}(D)} \\
& \leq \sum_{\boldsymbol{\beta}<\boldsymbol{\alpha}}\left(\begin{array}{c}
\boldsymbol{\alpha} \\
\boldsymbol{\beta}
\end{array}\right)|\boldsymbol{\alpha}-\boldsymbol{\beta}| ! c_{\mathbf{A}} \boldsymbol{\mu}^{\boldsymbol{\alpha}-\boldsymbol{\beta}}\left\|\partial_{\mathbf{y}}^{\boldsymbol{\beta}} u(\mathbf{y})\right\|_{H^{1}(D)}\left\|\partial_{\mathbf{y}}^{\boldsymbol{\alpha}} u(\mathbf{y})\right\|_{H^{1}(D)},
\end{aligned}
$$

from which we derive

$$
\left\|\partial_{\mathbf{y}}^{\boldsymbol{\alpha}} u(\mathbf{y})\right\|_{H^{1}(D)} \leq \frac{c}{2} \sum_{\boldsymbol{\beta}<\boldsymbol{\alpha}}\left(\begin{array}{c}
\boldsymbol{\alpha} \\
\boldsymbol{\beta}
\end{array}\right)|\boldsymbol{\alpha}-\boldsymbol{\beta}| ! \boldsymbol{\mu}^{\boldsymbol{\alpha}-\boldsymbol{\beta}}\left\|\partial_{\mathbf{y}}^{\boldsymbol{\beta}} u(\mathbf{y})\right\|_{H^{1}(D)},
$$

where

$$
c:=\frac{a_{\max }}{a_{\min } c_{V}^{2}} \max \left\{2 c_{\mathbf{A}},\|f\|_{\widetilde{H}^{-1}(D)}+\|g\|_{H^{-1 / 2}\left(\Gamma_{N}\right)}\right\} .
$$

We note that, by the definition of $c$, we have $c \geq 2$ and, furthermore, because of Lemma 3.2, we also have that $\|u(\mathbf{y})\|_{H^{1}(D)} \leq c$, which means that the assertion is true for $|\boldsymbol{\alpha}|=0$.

Thus, we can use an induction over $|\boldsymbol{\alpha}|$ to prove the hypothesis

$$
\left\|\partial_{\mathbf{y}}^{\boldsymbol{\alpha}} u(\mathbf{y})\right\|_{H^{1}(D)} \leq|\boldsymbol{\alpha}| ! \boldsymbol{\mu}^{\boldsymbol{\alpha}} c^{|\boldsymbol{\alpha}|+1}
$$

Copyright $@$ by SIAM. Unauthorized reproduction of this article is prohibited. 
for $|\boldsymbol{\alpha}|>0$. Let the assertions hold for all $\boldsymbol{\alpha}$ which satisfy $|\boldsymbol{\alpha}| \leq n-1$ for some $n \geq 1$. Then, we know for all $\boldsymbol{\alpha}$ with $|\boldsymbol{\alpha}|=n$ that

$$
\begin{aligned}
\left\|\partial_{\mathbf{y}}^{\boldsymbol{\alpha}} u(\mathbf{y})\right\|_{H^{1}(D)} & \leq \frac{c}{2} \sum_{\boldsymbol{\beta}<\boldsymbol{\alpha}}\left(\begin{array}{c}
\boldsymbol{\alpha} \\
\boldsymbol{\beta}
\end{array}\right)|\boldsymbol{\alpha}-\boldsymbol{\beta}| ! \boldsymbol{\mu}^{\boldsymbol{\alpha}-\boldsymbol{\beta}}\left\|\partial_{\mathbf{y}}^{\boldsymbol{\beta}} u(\mathbf{y})\right\|_{H^{1}(D)} \\
& \leq \frac{c}{2} \boldsymbol{\mu}^{\boldsymbol{\alpha}} \sum_{\boldsymbol{\beta}<\boldsymbol{\alpha}}\left(\begin{array}{c}
\boldsymbol{\alpha} \\
\boldsymbol{\beta}
\end{array}\right)|\boldsymbol{\alpha}-\boldsymbol{\beta}| !|\boldsymbol{\beta}| ! c^{|\boldsymbol{\beta}|+1} \\
& =\frac{c}{2} \boldsymbol{\mu}^{\boldsymbol{\alpha}} \sum_{j=0}^{n-1} \sum_{\substack{\boldsymbol{\beta}<\boldsymbol{\alpha} \\
|\boldsymbol{\beta}|=j}}\left(\begin{array}{c}
\boldsymbol{\alpha} \\
\boldsymbol{\beta}
\end{array}\right)|\boldsymbol{\alpha}-\boldsymbol{\beta}| !|\boldsymbol{\beta}| ! c^{|\boldsymbol{\beta}|+1}
\end{aligned}
$$

Making use of the combinatorial identity (15) yields

$$
\begin{aligned}
\left\|\partial_{\mathbf{y}}^{\boldsymbol{\alpha}} u(\mathbf{y})\right\|_{H^{1}(D)} & \leq \frac{c}{2} \boldsymbol{\mu}^{\boldsymbol{\alpha}} \sum_{j=0}^{n-1}\left(\begin{array}{c}
|\boldsymbol{\alpha}| \\
j
\end{array}\right)(|\boldsymbol{\alpha}|-j) ! j ! c^{j+1} \\
& =\frac{c}{2}|\boldsymbol{\alpha}| ! \boldsymbol{\mu}^{\boldsymbol{\alpha}} c \sum_{j=0}^{n-1} c^{j} \leq \frac{c}{2}|\boldsymbol{\alpha}| ! \boldsymbol{\mu}^{\boldsymbol{\alpha}} c \frac{c^{|\boldsymbol{\alpha}|}}{c-1} \leq \frac{c}{2(c-1)}|\boldsymbol{\alpha}| ! \boldsymbol{\mu}^{\boldsymbol{\alpha}} c^{|\boldsymbol{\alpha}|+1} .
\end{aligned}
$$

Now, since $c \geq 2$, we have $c \leq 2(c-1)$ and, hence, also

$$
\left\|\partial_{\mathbf{y}}^{\boldsymbol{\alpha}} u(\mathbf{y})\right\|_{H^{1}(D)} \leq|\boldsymbol{\alpha}| ! \boldsymbol{\mu}^{\boldsymbol{\alpha}} c^{|\boldsymbol{\alpha}|+1} .
$$

This completes the proof.

4.3. Numerical quadrature in the parameter. Because of the regularity estimates shown before, we can refer to [13, Lemma 7], which is a straightforward consequence from the results in [21], for the convergence rate of the quasi-Monte Carlo method (QMC) based on the Halton points. Therefore, assuming that there is an $\varepsilon>0$ such that $\gamma_{k} \leq c k^{-3-\varepsilon}$ for some constant $c>0$, we can conclude that, for any $\delta>0$, there is a constant $C_{\delta}$ such that

$$
\left\|\mathbb{E}[u]-\frac{1}{N} \sum_{i=1}^{N} u\left(2 \boldsymbol{\xi}_{i}-1\right)\right\|_{H^{1}(D)} \leq C_{\delta} N^{\delta-1} .
$$

A similar result also accounts for the variance $\mathbb{V}[u]$; see, e.g., [14].

For the sparse grid (SG) quadrature, assume that $\gamma_{k} \leq c k^{-r-1}$ for some constants $c, r>0$. Then, the anisotropic sparse Gauss-Legendre quadrature on level $q$ with $N(q)$ points satisfies the error estimate

$$
\left\|\mathbb{E}[u]-\mathcal{A}_{\mathbf{w}}(q, M) u\right\|_{H_{0}^{1}(D)} \leq C N(q)^{-r /(2 \log \log M)}\|u\|_{C\left(\square ; H_{0}^{1}(D)\right)}
$$

with a constant $C>0$; see [10]. Herein, we have $w_{k}:=\log \left(\frac{1}{k \gamma_{k}}+\sqrt{1+1 /\left(k \gamma_{k}\right)^{2}}\right)$ (see, e.g., [4]), and

$$
\mathcal{A}_{\mathbf{w}}(q, M):=\sum_{\boldsymbol{\alpha} \in Y_{\mathbf{w}}(q, M)} c_{\mathbf{w}}(\boldsymbol{\alpha}) \mathbf{Q}_{\boldsymbol{\alpha}} \quad \text { with } \quad c_{\mathbf{w}}(\boldsymbol{\alpha}):=\sum_{\substack{\boldsymbol{\beta} \in\{0,1\} M \\\langle\boldsymbol{\alpha}+\boldsymbol{\beta}, \mathbf{w}\rangle \leq q}}(-1)^{|\boldsymbol{\beta}|},
$$

Copyright $@$ by SIAM. Unauthorized reproduction of this article is prohibited. 
where $\mathbf{Q}_{\boldsymbol{\alpha}}$ denotes the tensor product Gauss-Legendre quadrature operator of degree $\lceil\boldsymbol{\alpha} / 2\rceil .{ }^{3}$ The set $Y_{\mathbf{w}}(q, M)$ is given according to

$$
Y_{\mathbf{w}}(q, M):=\left\{\boldsymbol{\alpha} \in \mathbb{N}_{0}^{M}: q-\|\mathbf{w}\|_{1} \leq\langle\boldsymbol{\alpha}, \mathbf{w}\rangle \leq q\right\} .
$$

With similiar arguments as in [14], the convergence result again carries over to $\mathbb{V}[u]$.

5. Numerical results. We will now consider two examples of the model problem (1) with a diffusion coefficient of form (3) using the unit cube $D:=(0,1)^{3}$ as the domain of computation. In both examples, we set the global strength $a$ to $a:=0.12$ and, for convenience, segment the boundary $\partial D$ into three disjoint parts:

$$
\begin{aligned}
& \Gamma_{0}:=\{1\} \times(0,1) \times(0,1), \\
& \Gamma_{1}:=\{0\} \times(0,1) \times(0,1), \text { and } \\
& \Gamma_{2}:=\partial D \backslash\left(\Gamma_{0} \cup \Gamma_{1}\right) .
\end{aligned}
$$

Moreover, we choose the description of $\mathbf{V}$ to be defined by $\mathbb{E}[\mathbf{V}](\mathbf{x}):=\left[\begin{array}{lll}1 & 0 & 0\end{array}\right]^{\top}$ and

$$
\operatorname{Cov}[\mathbf{V}]\left(\mathbf{x}, \mathbf{x}^{\prime}\right):=0.01 \exp \left(-\frac{\left\|\mathbf{x}-\mathbf{x}^{\prime}\right\|_{2}^{2}}{50}\right)\left[\begin{array}{ccc}
1 & 0 & 0 \\
0 & 9 s_{2}\left(\mathbf{x}, \mathbf{x}^{\prime}\right) & 0 \\
0 & 0 & 9 s_{3}\left(\mathbf{x}, \mathbf{x}^{\prime}\right)
\end{array}\right]
$$

where

$$
s_{j}\left(\mathbf{x}, \mathbf{x}^{\prime}\right):=16 \cdot x_{j}\left(1-x_{j}\right) \cdot x_{j}^{\prime}\left(1-x_{j}^{\prime}\right) .
$$

The effect of the function $s_{j}$ is to suppress the covariance along the normal direction on the boundary $\Gamma_{2}$. Some samples of the normalized vector field $\mathbf{V} /\|\mathbf{V}\|_{2}$ used for our examples, which are computed on the level 3 discretization, are shown in Figure 8 as stream traces. By their definition, the stream traces are tracing our notable diffusion direction.

The numerical implementation is performed with aid of the problem-solving environment DOLFIN [17], which is a part of the FEniCS Project [17]. The KarhunenLoève expansion of the vector field $\mathbf{V}$ is computed by the pivoted Cholesky decomposition; see $[11,12]$ for the details. For the finite element discretization, we employ a sequence of triangulations $\mathcal{T}_{l}$; subsequently, we will call the index $l$ the level, yielded by successive uniform refinement, i.e., cutting each tetrahedron into 8 tetrahedra. Level 0 consists of $6 \cdot 2^{3}=48$ tetrahedra. Then, we use elementwise constant functions and the truncated pivoted Cholesky decomposition for the Karhunen-Loève expansion approximation and continuous elementwise linear functions in space. The truncation criterion for the pivoted Cholesky decomposition is that the relative trace error is smaller than $10^{-4} \cdot 4^{-l}$; see Table 1 for the resulting parameter dimensions $M$. Since the exact solutions of the examples are unknown, the errors will have to be estimated. Therefore, in this section, we will estimate the errors for levels 0 to 5 by substituting the exact solution with the approximate solution computed on the level 6 triangulation $\mathcal{T}_{6}$ using the QMC quadrature based on Halton points with $10^{4}$ samples; e.g., we approximate the error for the mean according to

\footnotetext{
${ }^{3}$ Note that the quadrature operator $\mathcal{A}_{\mathbf{w}}(q, M)$ refers rather to the SG combination technique than the actual SG quadrature operator.
} 
TABLE 1

The number of samples for the first six levels and the respective parameter dimensions.

\begin{tabular}{lrrrrrr}
\hline$l$ & 0 & 1 & 2 & 3 & 4 & 5 \\
\hline$N_{l}^{\mathrm{QMC}}$ & 10 & 24 & 57 & 135 & 320 & 762 \\
$N_{l}^{\mathrm{SG}}$ & 1 & 7 & 29 & 87 & 265 & 909 \\
$N_{l}^{\mathrm{MC}}$ & 1 & 4 & 16 & 64 & 256 & 1024 \\
\hline$M$ & 16 & 24 & 28 & 34 & 44 & 53 \\
\hline
\end{tabular}

$$
\begin{aligned}
\| \mathbb{E}[u] & -\frac{1}{N_{l}^{\mathrm{QMC}}} \sum_{i=1}^{N_{l}^{\mathrm{QMC}}} u_{l}\left(2 \boldsymbol{\xi}_{i}-1\right) \|_{H^{1}(D)} \\
\approx & \left\|\frac{1}{10^{4}} \sum_{i=1}^{10^{4}} u_{6}\left(2 \boldsymbol{\xi}_{i}-1\right)-\frac{1}{N_{l}^{\mathrm{QMC}}} \sum_{i=1}^{N_{l}^{\mathrm{QMC}}} u_{l}\left(2 \boldsymbol{\xi}_{i}-1\right)\right\|_{H^{1}(D)}
\end{aligned}
$$

and

$$
\left\|\mathbb{E}[u]-\mathcal{A}_{\mathbf{w}}\left(q_{l}, M\right) u_{l}\right\|_{H^{1}(D)} \approx\left\|\frac{1}{10^{4}} \sum_{i=1}^{10^{4}} u_{6}\left(2 \boldsymbol{\xi}_{i}-1\right)-\mathcal{A}_{\mathbf{w}}\left(q_{l}, M\right) u_{l}\right\|_{H^{1}(D)},
$$

where $u_{l}$ is the finite element approximation on the triangulation $\mathcal{T}_{l}$.

For every level, we also define the number of samples used by the different quadrature methods. For the QMC method based on Halton points, we choose

$$
N_{l}^{\mathrm{QMC}}:=\left\lceil 2^{l /(1-\delta)} \cdot 10\right\rceil
$$

with $\delta:=0.2$; see Table 1 for the resulting values of $N_{l}$. For the SG quadrature, we set $q_{l}=2 l+2$. Based on these choices, we expect to see an asymptotic rate of convergence of $2^{-l}$ in the $H^{1}$-norm for the mean and in the $W^{1,1}$-norm for the variance; see, again, Table 1 for the resulting values of $N_{l}^{\mathrm{SG}}:=N\left(q_{l}\right)$. As a validation for the reference solution, we consider here also the convergence of a Monte Carlo (MC) quadrature, using $N_{l}^{\mathrm{MC}}:=4^{l}$ samples on the level $l$, with respect to this reference. Note that to obtain an approximation for the mean square error, we average five realizations of the MC estimator.

5.1. Example. In the first example, we set the source to $f(\mathbf{x}) \equiv 1$ and consider homogeneous Dirichlet data, i.e., we set $\Gamma_{D}=\Gamma_{0} \cup \Gamma_{1} \cup \Gamma_{2}$ and $\Gamma_{N}=\emptyset$.

Visualizations of the reference solution's mean and variance are shown in Figure 2. Note that, to enable a view of the inside, all data with coordinates $\left[x_{1}, x_{2}, x_{3}\right]^{\top}$ such that $x_{2}+x_{3}>1$ are clipped.

Figures 3 and 4 show the estimated errors of the solution's mean on the left-hand side and of the solution's variance on the right-hand side, each versus the discretization level for the different quadrature methods. As expected, each of the quadrature methods achieves the predicted rate of convergence; however, QMC and SG provide slightly better errors in the case of the variance.

5.2. Example. The data in this example are given as follows. We remove the source, i.e., $f(\mathbf{x}) \equiv 0$, and consider 

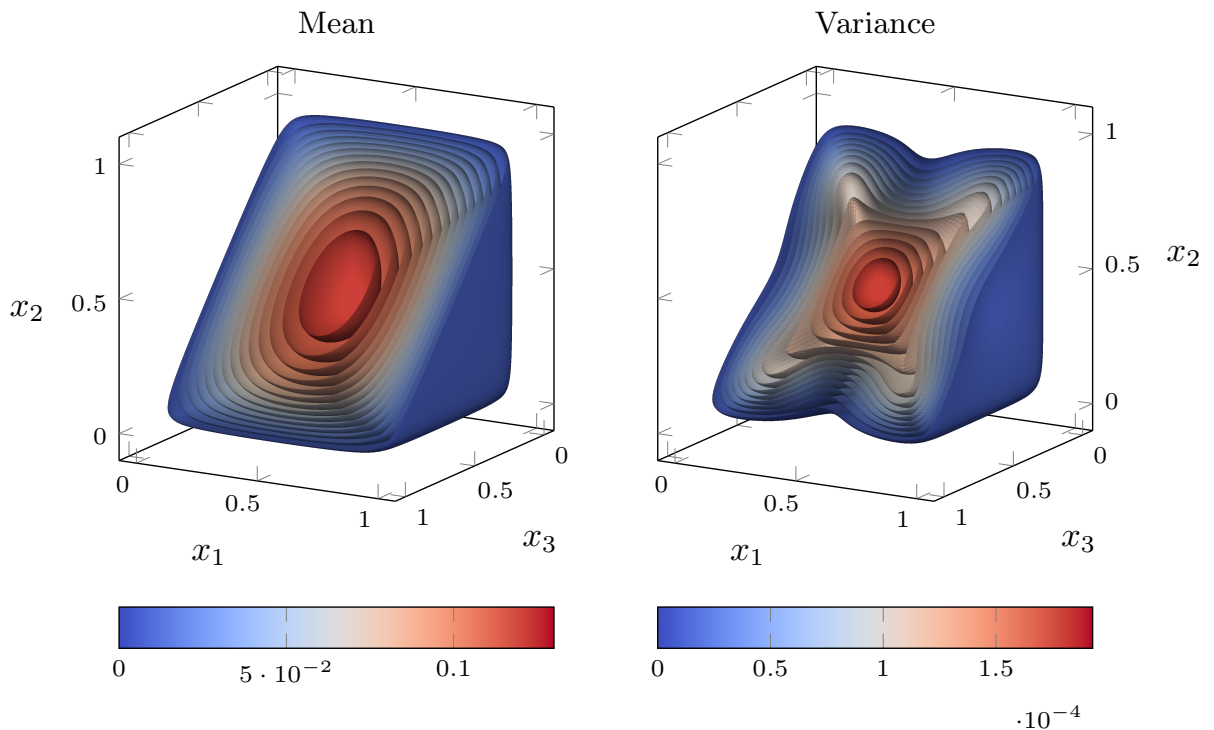

FIG. 2. Mean and variance of the solution.

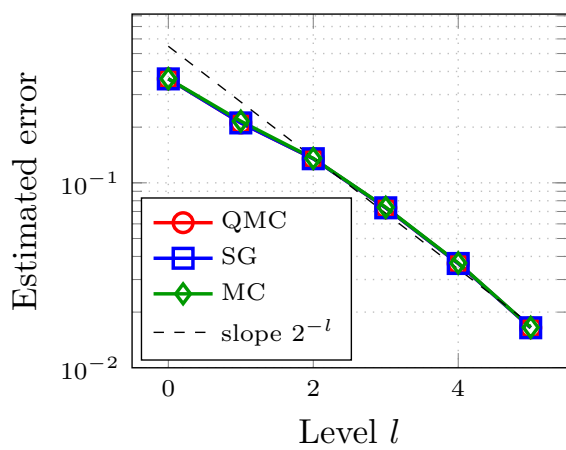

FIG. 3. $H^{1}$-error in the mean.

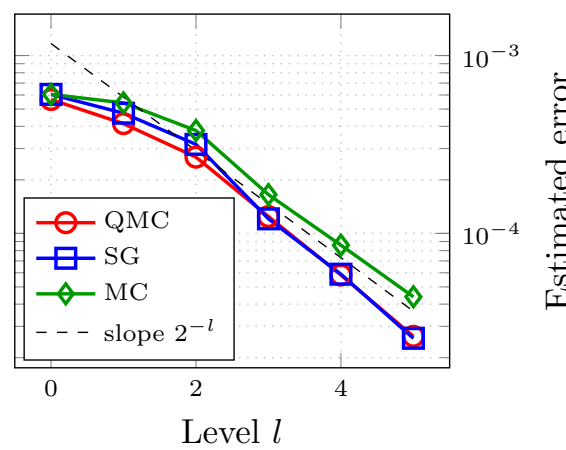

FIG. 4. $W^{1,1}$-error in the variance.

$$
\Gamma_{N}:=\Gamma_{0} \cup \Gamma_{1} \quad \text { with } \quad g(\mathbf{x}):= \begin{cases}1, & \mathbf{x} \in \Gamma_{0}, \\ -1, & \mathbf{x} \in \Gamma_{1},\end{cases}
$$

and $\Gamma_{D}:=\Gamma_{2}$.

The respective visualizations of the reference solution's mean and variance are depicted in Figure 5.

Figures 6 and 7 exhibit the estimated errors of the solution's mean on the left-hand side and of the solution's variance on the right-hand side, each versus the discretization level for the different quadrature methods. Again, each of the quadrature methods achieves the predicted rate of convergence. As in the previous example, QMC and SG provide slightly better errors in the case of the variance.

6. Conclusion. In this article, we have introduced the diffusion coefficient (3) that may be used to model anisotropic diffusion that has a notable direction of diffusion with an associated strength, both of which are considered to be subject to 

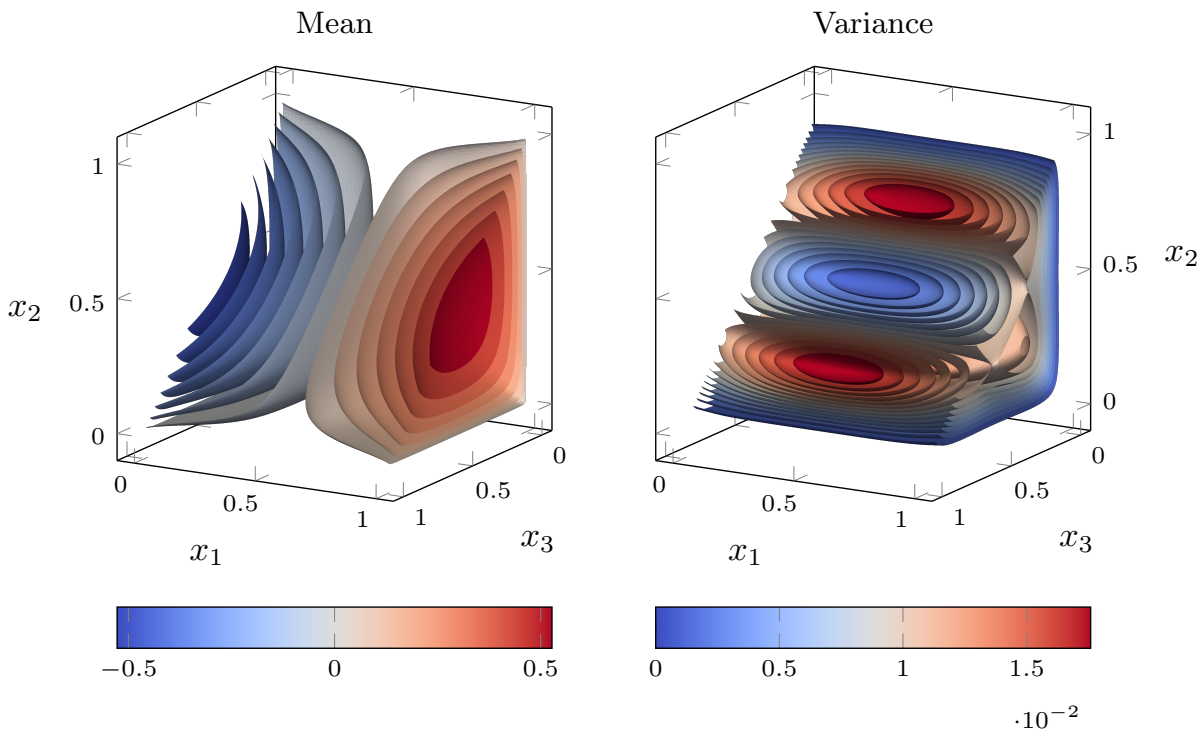

FIG. 5. Mean and variance of the solution.

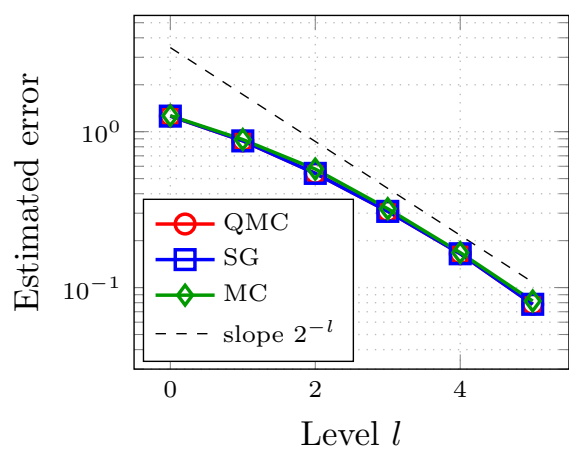

FIG. 6. $H^{1}$-error in the mean.

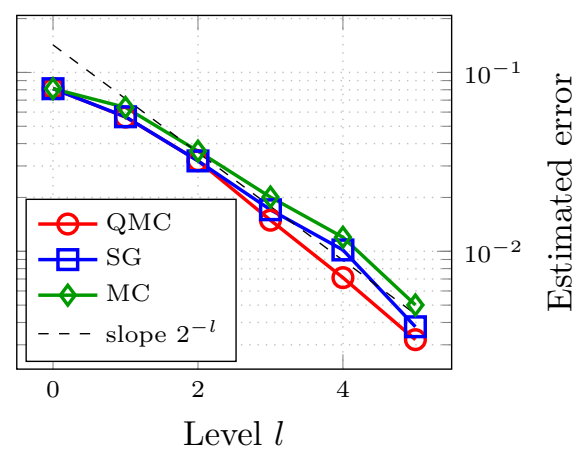

FIG. 7. $W^{1,1}$-error in the variance.

uncertainty; this is encoded by the vector field $\mathbf{V}$. While this type of diffusion coefficient does not model all possible anisotropic diffusion coefficients, it can be used to model both diffusion in media that consist of thin fibers or thin sheets, given that either the diffusion between the fibers or in the sheets is isotropic with a global strength that is not subject to uncertainty.

We derived, based on the decay of the Karhunen-Loève expansion of $\mathbf{V}$, related decay rates for the solution's derivatives; given a sufficiently fast decaying KarhunenLoève expansion, this regularity then provides dimension independent convergence when considering the QMC quadrature to approximate quantities of interest that require the integration of the solution with respect to the random parameter. Furthermore, it also allows the use of other quadrature methods like the anisotropic SG quadrature which has been considered in the numerical experiments. The numerical results corroborate the theoretical findings. 

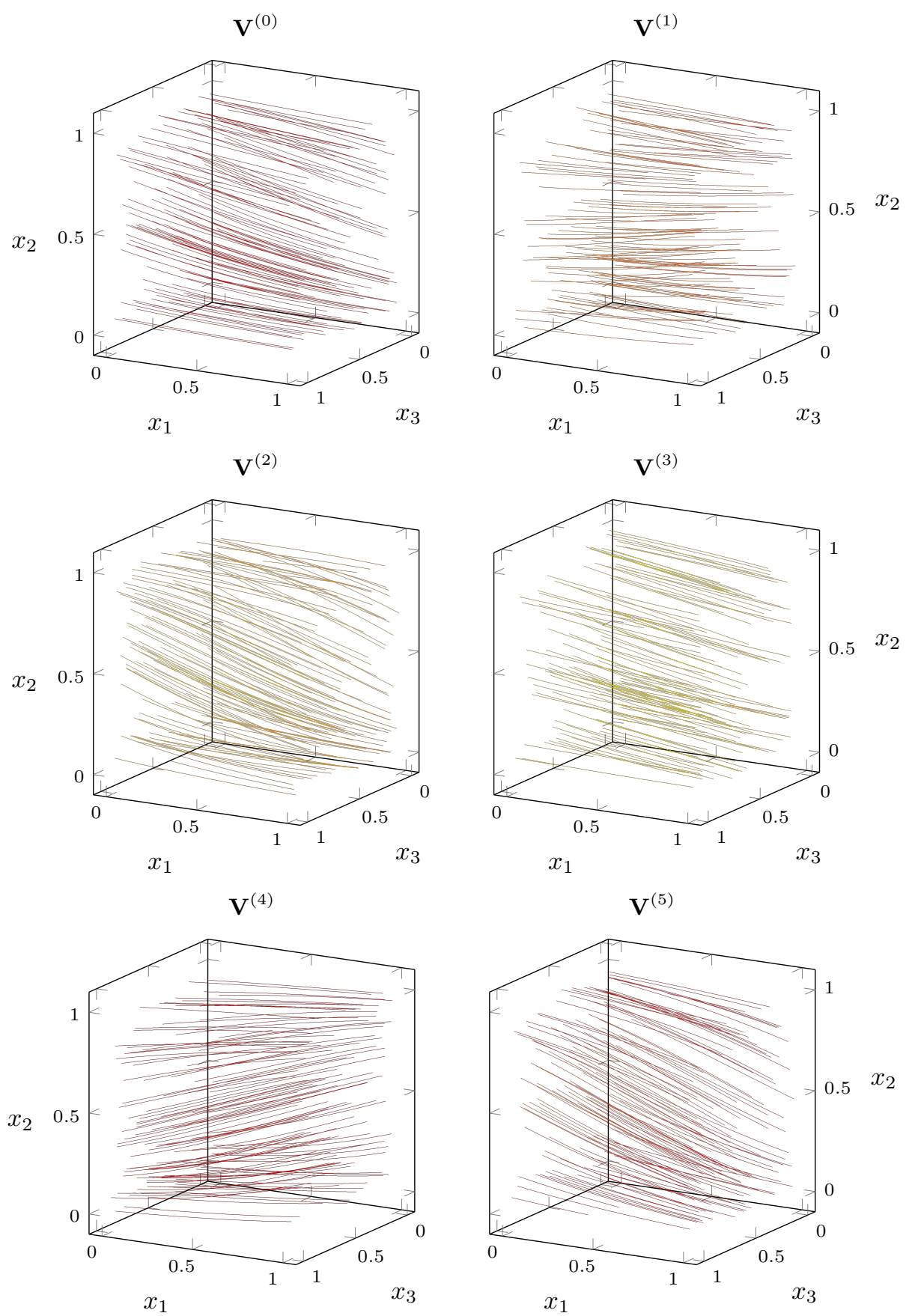

FIG. 8. Stream traces of some samples of the vector field $\mathbf{V} /\|\mathbf{V}\|_{2}$ used for the examples.

Copyright (c) by SIAM. Unauthorized reproduction of this article is prohibited. 
Last, note that the model for the diffusion coefficient may, for example, be generalized to

$$
\mathbf{A}(\mathbf{x}, \omega):=a(\mathbf{x}, \omega) \mathbf{I}+\left(\|\mathbf{V}(\mathbf{x}, \omega)\|_{2}-a(\mathbf{x}, \omega)\right) \frac{\mathbf{V}(\mathbf{x}, \omega) \mathbf{V}^{\top}(\mathbf{x}, \omega)}{\mathbf{V}^{\top}(\mathbf{x}, \omega) \mathbf{V}(\mathbf{x}, \omega)}
$$

without affecting regularity. With this type of diffusion coefficient, the diffusion perpendicular to the fibers is still isotropic at any point in the domain. However, it may depend on the particular location and also be subjected to uncertainty. We especially note that for two spatial dimensions this generalization already models all types of random anisotropic diffusion coefficients.

\section{Appendix.}

Lemma A.1. We have $\operatorname{img}(\mathcal{C}) \subset L^{\infty}\left(D ; \mathbb{R}^{d}\right)$. This implies that $\boldsymbol{\psi}_{k} \in L^{\infty}\left(D ; \mathbb{R}^{d}\right)$ and, as a consequence, also $Y_{k}(\omega) \in L_{\mathbb{P}}^{\infty}(\Omega)$.

Proof. For almost every $\mathbf{x} \in D$, we know that $\operatorname{Cov}[\mathbf{V}](\mathbf{x}, \cdot) \in L^{\infty}\left(D ; \mathbb{R}^{d \times d}\right)$, where we clearly have

$$
\|\operatorname{Cov}[\mathbf{V}](\mathbf{x}, \cdot)\|_{L^{\infty}\left(D ; \mathbb{R}^{d \times d}\right)} \leq\|\operatorname{Cov}[\mathbf{V}]\|_{L^{\infty}\left(D ; L^{\infty}\left(D ; \mathbb{R}^{d \times d}\right)\right)} .
$$

Thus, we can calculate for almost every $\mathbf{x} \in D$ that

$$
\begin{aligned}
\|(\mathcal{C} \mathbf{u})(\mathbf{x})\|_{2} & \leq \int_{D}\left\|\operatorname{Cov}[\mathbf{V}]\left(\mathbf{x}, \mathbf{x}^{\prime}\right) \mathbf{u}\left(\mathbf{x}^{\prime}\right)\right\|_{2} \mathrm{~d} \mathbf{x}^{\prime} \\
& \leq \int_{D}\left\|\operatorname{Cov}[\mathbf{V}]\left(\mathbf{x}, \mathbf{x}^{\prime}\right)\right\|_{F}\left\|\mathbf{u}\left(\mathbf{x}^{\prime}\right)\right\|_{2} \mathrm{~d} \mathbf{x}^{\prime} \\
& \leq\|\operatorname{Cov}[\mathbf{V}]\|_{L^{\infty}\left(D ; L^{\infty}\left(D ; \mathbb{R}^{d \times d}\right)\right)} \sqrt{|D|}\|\mathbf{u}\|_{L^{2}\left(D ; \mathbb{R}^{d}\right)}
\end{aligned}
$$

and conclude that $\|\mathcal{C} \mathbf{u}\|_{L^{\infty}\left(D ; \mathbb{R}^{d}\right)} \leq\|\operatorname{Cov}[\mathbf{V}]\|_{L^{\infty}\left(D ; L^{\infty}\left(D ; \mathbb{R}^{d \times d}\right)\right)} \sqrt{|D|}\|\mathbf{u}\|_{L^{2}\left(D ; \mathbb{R}^{d}\right)}$.

Lemma A.2. The representation (6) also converges in $L_{\mathbb{P}}^{\infty}\left(\Omega ; L^{\infty}\left(D ; \mathbb{R}^{d}\right)\right)$.

Proof. We define

$$
\mathbf{V}^{M}(\mathbf{x}, \mathbf{y}):=\mathbb{E}[\mathbf{V}](\mathbf{x})+\sum_{k=1}^{M} \sigma_{k} \boldsymbol{\psi}_{k}(\mathbf{x}) y_{k} .
$$

Since $L_{\mathbb{P}_{\mathbf{y}}}^{\infty}\left(\square ; L^{\infty}\left(D ; \mathbb{R}^{d}\right)\right)$ is complete, it suffices to show that $\left(\mathbf{V}^{M}\right)_{M \in \mathbb{N}}$ is a Cauchy sequence in $L_{\mathbb{P}_{\mathbf{y}}}^{\infty}\left(\square ; L^{\infty}\left(D ; \mathbb{R}^{d}\right)\right)$. Let $M \leq M^{\prime}$ be two indices; then we have

$$
\begin{aligned}
\left\|\mathbf{V}^{M^{\prime}}-\mathbf{V}^{M}\right\|_{L_{\mathbb{P}_{\mathbf{Y}}}^{\infty}\left(\square ; L^{\infty}\left(D ; \mathbb{R}^{d}\right)\right)} & =\left\|\sum_{k=M+1}^{M^{\prime}} \sigma_{k} \boldsymbol{\psi}_{k} y_{k}\right\|_{L_{\mathbb{P}_{\mathbf{Y}}}^{\infty}\left(\square ; L^{\infty}\left(D ; \mathbb{R}^{d}\right)\right)} \\
& \leq \sum_{k=M+1}^{M^{\prime}}\left\|\sigma_{k} \boldsymbol{\psi}_{k}\right\|_{L^{\infty}\left(D ; \mathbb{R}^{d}\right)}\left\|y_{k}\right\|_{L_{\mathbb{P}_{\mathbf{y}}}^{\infty}(\square ; \mathbb{R})} \\
& \leq \sum_{k=M+1}^{M^{\prime}} \gamma_{k} \leq \sum_{k=M+1}^{\infty} \gamma_{k} .
\end{aligned}
$$

Thus, since $\gamma \in \ell^{1}\left(\mathbb{N}_{0}\right)$, we know that $\left\|\mathbf{V}^{M^{\prime}}-\mathbf{V}^{M}\right\|_{L_{\mathbb{P}_{\mathbf{Y}}}^{\infty}\left(\square ; L^{\infty}\left(D ; \mathbb{R}^{d}\right)\right)} \stackrel{M, M^{\prime} \rightarrow \infty}{\longrightarrow} 0$ and so $\left(\mathbf{V}^{M}\right)_{M \in \mathbb{N}}$ is a Cauchy sequence in $L_{\mathbb{P}_{\mathbf{y}}}^{\infty}\left(\square ; L^{\infty}\left(D ; \mathbb{R}^{d}\right)\right)$.

Copyright $@$ by SIAM. Unauthorized reproduction of this article is prohibited. 
Lemma A.3. The condition (4) is satisfied by any truncation of the KarhunenLoève expansion with a large enough $M$.

Proof. Recall the definition

$$
\mathbf{V}^{M}(\mathbf{x}, \mathbf{y}):=\mathbb{E}[\mathbf{V}](\mathbf{x})+\sum_{k=1}^{M} \sigma_{k} \boldsymbol{\psi}_{k}(\mathbf{x}) y_{k}
$$

Clearly, for any $M$ we have that

$$
\begin{aligned}
\left\|\mathbf{V}^{M}\right\|_{d} & =\left\|\mathbb{E}[\mathbf{V}]+\sum_{k=1}^{M} \sigma_{k} \boldsymbol{\psi}_{k} y_{k}\right\|_{d}=\left\|\boldsymbol{\psi}_{0}+\sum_{k=1}^{M} \sigma_{k} \boldsymbol{\psi}_{k} y_{k}\right\| \|_{d} \\
& \leq\left\|\boldsymbol{\psi}_{0}\right\|_{d}+\sum_{k=1}^{M}\left\|\sigma_{k} \boldsymbol{\psi}_{k} y_{k}\right\|_{d} \leq \sum_{k=0}^{M} \gamma_{k} \leq \sum_{k=0}^{\infty} \gamma_{k}<\infty
\end{aligned}
$$

since $\gamma \in \ell^{1}\left(\mathbb{N}_{0}\right)$. Then, because of

$$
\begin{aligned}
\underset{\mathbf{y} \in \square}{\operatorname{essinf}} \underset{\mathbf{x} \in D}{\operatorname{essinf}}\left\|\mathbf{V}^{M}(\mathbf{x}, \mathbf{y})\right\|_{2} & =\underset{\mathbf{y} \in \square}{\operatorname{essinf}} \underset{\mathbf{x} \in D}{\operatorname{essinf}}\left\|\mathbb{E}[\mathbf{V}](\mathbf{x})+\sum_{k=1}^{M} \sigma_{k} \boldsymbol{\psi}_{k}(\mathbf{x}) y_{k}\right\|_{2} \\
& =\underset{\mathbf{y} \in \square}{\operatorname{essinf}} \underset{\mathbf{x} \in D}{\operatorname{essinf}}\left\|\mathbf{V}(\mathbf{x}, \mathbf{y})-\sum_{k=M+1}^{\infty} \sigma_{k} \boldsymbol{\psi}_{k}(\mathbf{x}) y_{k}\right\|_{2} \\
& \geq \underset{\mathbf{y} \in \square}{\operatorname{essinf}} \underset{\mathbf{x} \in D}{\operatorname{essinf}}\|\mathbf{V}\|_{2}-\left\|\sum_{k=M+1}^{\infty} \sigma_{k} \boldsymbol{\psi}_{k}(\mathbf{x}) y_{k}\right\|_{d} \\
& \geq a_{\min }-\sum_{k=M+1}^{\infty} \gamma_{k}
\end{aligned}
$$

for any $M$ that fulfils $\sum_{k=M+1}^{\infty} \gamma_{k}<a_{\text {min }}$, we can find constants with which $\mathbf{V}^{M}$ satisfies the condition (4). Since $\gamma \in \ell^{1}\left(\mathbb{N}_{0}\right)$ implies that $\sum_{k=M+1}^{\infty} \gamma_{k} \stackrel{M \rightarrow \infty}{\longrightarrow} 0$, we see that $\sum_{k=M+1}^{\infty} \gamma_{k}<a_{\min }$ is fulfilled for sufficiently large $M$.

\section{REFERENCES}

[1] M. Abramowitz And I. A. Stegun, Handbook of Mathematical Functions: With Formulas, Graphs, and Mathematical Tables, Appl. Math. Ser. 55, Dover, N. Chemsford, MA, 1964.

[2] R. A. Adams, Sobolev Spaces, Academic Press, New York, 1975.

[3] I. BABušKa And P. Chatzipantelidis, On solving elliptic stochastic partial differential equations, Comput. Methods Appl. Mech. Engrg, 191 (2002), pp. 4093-4122.

[4] I. BabušKa, F. Nobile, And R. Tempone, A stochastic collocation method for elliptic partial differential equations with random input data, SIAM J. Numer. Anal., 45 (2007), pp. 1005-1034.

[5] J. Beck, R. Tempone, F. Nobile, and L. Tamellini, On the optimal polynomial approximation of stochastic PDEs by Galerkin and collocation methods, Math. Models Methods Appl. Sci., 22 (2012), pp. 1250-023.

[6] A. Cohen, R. DeVore, and C. Schwab, Convergence rates of best $N$-term Galerkin approximations for a class of elliptic sPDEs, Found. Comput. Math., 10 (2010), pp. 615-646.

[7] G. M. Constantine and T. H. Savits, A multivariate Fà̀ di Bruno formula with applications, Trans. Amer. Math. Soc., 248 (1996), pp. 503-520.

[8] P. Frauenfelder, C. Schwab, and R. Todor, Finite elements for elliptic problems with stochastic coefficients, Comput. Methods Appl. Mech. Engrg, 194 (2005), pp. 205-228.

[9] S. GöKtePe And E. KunL, Computational modeling of cardiac electrophysiology: A novel finite element approach, Internat. J. Numer. Methods Engrg, 79 (2009), pp. 156-178.

Copyright $@$ by SIAM. Unauthorized reproduction of this article is prohibited. 
[10] A.-L. Haji-Ali, H. Harbrecht, M. Peters, and M. Siebenmorgen, Novel results for the anisotropic sparse grid quadrature, preprint, arXiv:1509.05521v2, 2015.

[11] H. Harbrecht, M. Peters, and R. Schneider, On the low-rank approximation by the pivoted Cholesky decomposition, Applied Numer. Math., 62 (2012), pp. 28-440.

[12] H. Harbrecht, M. Peters, and M. Siebenmorgen, Efficient approximation of random fields for numerical applications, Numer. Linear Algebra Appl., 22 (2015), pp. 596-617.

[13] H. Harbrecht, M. Peters, and M. Siebenmorgen, Analysis of the domain mapping method for elliptic diffusion problems on random domains, Numer. Math., 134 (2016), pp. 823-856.

[14] H. Harbrecht, M. Peters, and M. Siebenmorgen, On the quasi-Monte Carlo method with Halton points for elliptic PDEs with log-normal diffusion, Math. Comp., 86 (2017), pp. $771-797$.

[15] E. Hille And R. S. Phillips, Functional Analysis and Semi-Groups, Amer. Math. Soc. Collog. Publ. 31, American Mathematical Society, Providence, RI, 1957.

[16] W. A. Light and E. W. Cheney, Approximation theory in tensor product spaces, Lecture Notes in Math. 1169, Springer, New York, 1985.

[17] A. Logg, K. -A. Mardal, and G. Wells, Automated Solution of Differential Equations by the Finite Element Method, Springer, 2012.

[18] H. Matthies AND A. Keese, Galerkin methods for linear and nonlinear elliptic stochastic partial differential equations, Comput. Methods Appl. Mech. Engrg., 194 (2005), pp. $1295-1331$.

[19] D. Rohmer, A. Sitek, And G. T. Gullberg, Reconstruction and visualization of fiber and laminar structure in the normal human heart from ex vivo diffusion tensor magnetic resonance imaging (dtmri) data, Investigat. Radiol., 42 (2007), pp. 777-789.

[20] M. Sermesant, R. Chabiniok, P. Chinchapatnam, T. Mansi, F. Billet, P. Moireau, J. M. Peyrat, K. Wong, J. Relan, K. Rhode, M. Ginks, P. Lambiase, H. Delingette, M. Sorine, C. A. Rinaldi, D. Chapelle, R. Razavi, and N. Ayache, Patient-specific electromechanical models of the heart for the prediction of pacing acute effects in CRT: A preliminary clinical validation, Med. Image Analy., 16 (2012), pp. 201-215.

[21] X. WANG, A constructive approach to strong tractability using quasi-Monte Carlo algorithms, J. Complexity, 18 (2002), pp. 683-701.

Copyright $@$ by SIAM. Unauthorized reproduction of this article is prohibited. 